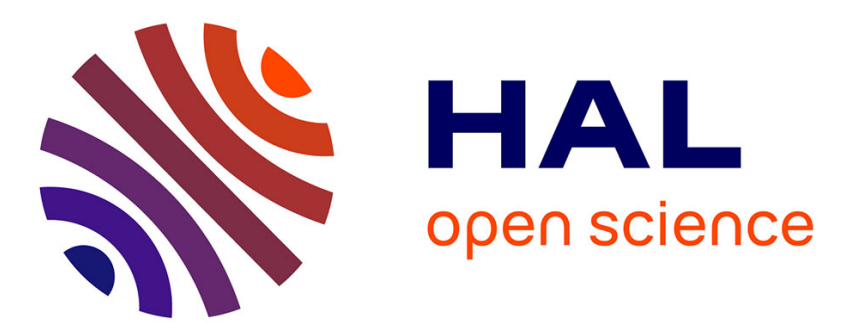

\title{
Modelling of a tuned liquid multi-column damper. Application to floating wind turbine for improved robustness against wave incidence
}

Christophe Coudurier, Olivier Lepreux, Nicolas Petit

\section{- To cite this version:}

Christophe Coudurier, Olivier Lepreux, Nicolas Petit. Modelling of a tuned liquid multi-column damper. Application to floating wind turbine for improved robustness against wave incidence. Ocean Engineering, 2018, 165, pp.277 - 292. 10.1016/j.oceaneng.2018.03.033 . hal-01865061

\section{HAL Id: hal-01865061}

https://hal-mines-paristech.archives-ouvertes.fr/hal-01865061

Submitted on 24 Oct 2018

HAL is a multi-disciplinary open access archive for the deposit and dissemination of scientific research documents, whether they are published or not. The documents may come from teaching and research institutions in France or abroad, or from public or private research centers.
L'archive ouverte pluridisciplinaire $\mathbf{H A L}$, est destinée au dépôt et à la diffusion de documents scientifiques de niveau recherche, publiés ou non, émanant des établissements d'enseignement et de recherche français ou étrangers, des laboratoires publics ou privés. 


\begin{abstract}
In this paper, the coupling of a float with a tuned liquid multi-column damper (TLMCD), a novel structural damping device inspired by the classical tuned liquid column damper (TLCD), is modelled using Lagrangian mechanics. We detail the tuning of the design parameters for each considered variant of the TLMCD, and compare each of them against a layout of multiple TLCDs. The results show that the proposed TLMCD is superior to multiple TLCDs for this application as it is more robust against wave incidence and it creates significantly less parasitic oscillations.
\end{abstract}

\title{
1. Introduction
}

Wind power is the second fastest growing source of renewable electricity [19] in terms of installed power. The construction of offshore wind farms is growing worldwide. In Europe, offshore wind energy is expected to grow to 23.5 GW by 2020, tripling the installed capacity in 2015 [5]. The major causes of this recent trend are the strength and regularity of wind far from the shore, which should allow for the easy mass production of electricity. To generate offshore wind energy, two types of technologies have been considered: fixedbottom wind turbines (foundations fixed into the seabed) and floating wind turbines (FWTs). The fixed-bottom offshore wind turbine technology is too costly for use in water deeper than $60 \mathrm{~m}$ [16]. This disqualifies them from use in most seas. FWTs are a tempting alternative. One advantage is that FWTs are not as dependent on seabed conditions for installation and can be moved to a harbour for maintenance. The main drawback of FWTs is their sensitivity to surrounding water waves that increase the mechanical load on the wind turbine [10], hence reducing the lifespan of its mechanical parts. This sensitivity can be mitigated by increasing the mass and size of the mechanical structure. However, this leads to a prohibitive rise in the cost per $\mathrm{kWh}$.

Previous studies have proposed compensating for tower fore-aft oscillations using collective and individual blade pitch control to modify the wind thrust forces $[10,17,2]$. This solution has the advantage of requiring no structural modification, but delivers limited performance. The tower movements are still 
many times superior to those observed on onshore wind turbines. Instead of using aerodynamic forces, it is tempting to consider using hydrodynamic forces. In naval engineering, considerable attention has been paid to ship roll damping (since the advent of steamboats). However, most solutions involve the use of the speed of the ship relative to the water to generate lift to control the roll [20] and, for this reason, are not easily transferable to our problem.

In addition to naval engineering, civil engineering has been a great contributor to such approaches, as skyscrapers are highly sensitive to wind gusts and earthquakes. This general field (structural control) is beyond the scope of this paper, and the reader can refer to [21] for an overview. To improve the response of massive structures to external disturbances, attached moving masses, such as tuned mass dampers (TMD), can be employed. Among the most economical and efficient solutions is the tuned liquid column damper (TLCD), also known as the anti-roll tank or the U-tank. As originally proposed by Frahm $[6,15]$ to limit ship roll, it is a U-shaped tube on a plane orthogonal to the ship's roll axis, and is generally filled with water. The liquid inside the TLCD oscillates due to the movement of the structure and liquid's energy is dissipated through a restriction located in the horizontal section. The TLCD is usually chosen to damp the natural frequency of the structure. While TLCD systems have been modelled in the past by, for instance, [1,7], it remains an active field of research [4]. A considerable amount of relevant research has been conducted over the last two decades on civil engineering applications, where most of the work has focused on determining the optimal design of passive TLCDs, such as [7, 25, 26].

Several studies have shown that the structural control of floating wind turbines using active $[11,18]$ or passive $[24,23]$ TMDs can substantially reduce the load on the wind turbine. Other studies $[3,14,22]$ have shown that the passive and semi-active TLCDs are an interesting alternative.

In this paper, we consider the damping of an offshore platform subject to waves of various angles of incidence. Such a system behaves as a six-DOF periodically oscillating rigid body. We try to minimize the roll and pitch oscillations by means of a TLCD, and neglect aerodynamic forces. Due to the mooring system, we cannot easily change the orientation of the float to adapt to the wave incidence. In the past, we studied the disturbance rejection capabilities of a TLCD aligned with the wave incidence [3]. As shown in Fig. 1, the damping provided by the TLCD is not robust against a change in the wave incidence.

This work is partly based on [8]. However, unlike the ships considered there, the float we consider has isotropic properties, meaning that its roll and pitch motions have the same characteristics. Here we go a step further introducing three multidirectional damping devices based on the concept of the TLCD. Their dynamics and their robustness against wave incidence are investigated.

\section{Description of the system}

The floater considered was the MIT/NREL Shallow Drafted Barge and the wind turbine was an NREL $5 \mathrm{MW}$; both are described in Tables 2 and 3. The barge and the wind turbine are modelled as a single rigid body, referred to as "the float" in this paper. Deformations in the wind turbine are neglected as its resonant period is inferior to the period of the monochromatic waves we consider here - ranging from $3 \mathrm{~s}$ to $30 \mathrm{~s}$. The float is studied with all six degrees of freedom. To avoid any bias in the study, we do not consider the interaction 


\begin{tabular}{|c|c|}
\hline $\mathcal{R}_{n}$ & Earth-fixed frame \\
\hline $\mathcal{R}_{b}$ & Barge-fixed frame \\
\hline$R(\Theta) \in \mathbb{R}^{3 \times 3}$ & $\begin{array}{l}\text { Rotation matrix from } \mathcal{R}_{b} \text { to } \mathcal{R}_{n} \\
\quad \text { so that } \forall r \in \mathbb{R}^{3}, r^{n}=R r^{b}\end{array}$ \\
\hline$x^{n}=[x, y, z]^{\top} \in \mathbb{R}^{3}$ & Position of the centre of gravity of barge in $\mathcal{R}_{n}$ \\
\hline$\Theta=[\varphi, \theta, \psi]^{\top} \in \mathbb{R}^{3}$ & Euler triple associated with $R$ \\
\hline$v^{b} \in \mathbb{R}^{3}$ & Speed of $C o G$, the centre of gravity of the float \\
\hline$\omega^{b} \in \mathbb{R}^{3}$ & Rotational speed of $\mathcal{R}_{b}$ with respect to $\mathcal{R}_{n}$ \\
\hline$n c$ & $\begin{array}{l}\text { Number of variables needed to describe } \\
\text { the liquid speed in the TLCD/TLMCD }\end{array}$ \\
\hline$w \in \mathbb{R}^{n c}$ & $\begin{array}{l}\text { Vector describing the position of } \\
\text { the liquid in the TLMCD }\end{array}$ \\
\hline$w_{i} \in \mathbb{R}$ & position of the liquid in the $i^{t h}$ element \\
\hline$q=\left[x^{n \top}, \Theta^{\top}, w^{\top}\right]^{\top} \in \mathbb{R}^{6+n c}$ & System's generalized positions \\
\hline$v=\left[v^{b \top}, \omega^{b \top}, \dot{w}^{\top}\right]^{\top} \in \mathbb{R}^{6+n c}$ & System's speeds \\
\hline$G(\Theta) \in \mathbb{R}^{3 \times 3}$ & Matrix relating $\dot{\Theta}$ and $\omega^{b}$ so that $\omega^{b}=G \dot{\Theta}$ \\
\hline $\mathcal{P}(\Theta) \in \mathbb{R}^{6+n c \times 6+n c}$ & Matrix relating $\dot{q}$ and $v$ so that $v=\mathcal{P} \dot{q}$ \\
\hline $\begin{array}{c}S(\cdot) \in \mathbb{R}^{3 \times 3} \\
S^{2}(\cdot)=S(\cdot)^{\top} S(\cdot)\end{array}$ & $\begin{array}{l}\text { Skew symmetric matrix representing the } \\
\text { cross-product in } \mathbb{R}^{3} \text {, with } S(x) y=x \times y \text {. }\end{array}$ \\
\hline$A_{v}$ and $A_{h} \in \mathbb{R}$ & $\begin{array}{c}\text { Cross-sections of the vertical and } \\
\text { horizontal tubes of the tank }\end{array}$ \\
\hline$\nu \in \mathbb{R}$ & Cross-section ratio defined as $\nu \triangleq A_{v} / A_{h}$ \\
\hline$\sigma_{i} \in \mathbb{R}$ & Curvilinear abscissa describing the geometry of the $i^{t h}$ element \\
\hline$\varsigma_{i}, \varsigma_{p i}, \varsigma_{s i} \in \mathbb{R}$ & Abscissa of the free surfaces in the $i^{t h}$ element \\
\hline$\alpha_{i} \in \mathbb{R}$ & orientation angle of the $i^{\text {th }}$ element \\
\hline$r^{b}(\sigma)=\left[x_{t}^{b}, y_{t}^{b}(\sigma), z_{t}^{b}(\sigma)\right]^{\top} \in \mathbb{R}^{3}$ & Function describing the centreline of the damper \\
\hline$A(\sigma)>0 \in \mathbb{R}$ & Cross-section of the tank at abscissa $\sigma$ \\
\hline$L_{v}$ and $L_{h} \in \mathbb{R}$ & Length of the vertical and horizontal tubes of the TLCD \\
\hline$e \in \mathbb{R}$ & Distance between $C o G$ and the horizontal tubes \\
\hline$\rho \in \mathbb{R}$ & Liquid density \\
\hline$\eta \in \mathbb{R}^{n c}$ & Vector of the head-loss coefficients of the restrictions \\
\hline$M_{s}=M_{s}^{\top} \in \mathbb{R}^{6 \times 6}$ & Mass matrix of the float \\
\hline$m_{t} \in \mathbb{R}$ & Total mass of the liquid in the damping system \\
\hline$Q_{\text {hydro }} \in \mathbb{R}^{6}$ & Generalized force due to the barge/waves interactions \\
\hline$Q_{r e s} \in \mathbb{R}^{n c}$ & Generalized force due to the restrictions in the TLMCD \\
\hline$F_{h} \in \mathbb{R}^{N}$ & Force generated by the fluid flow through the restrictions \\
\hline$\beta \in \mathbb{R}$ & Wave incidence angle \\
\hline
\end{tabular}




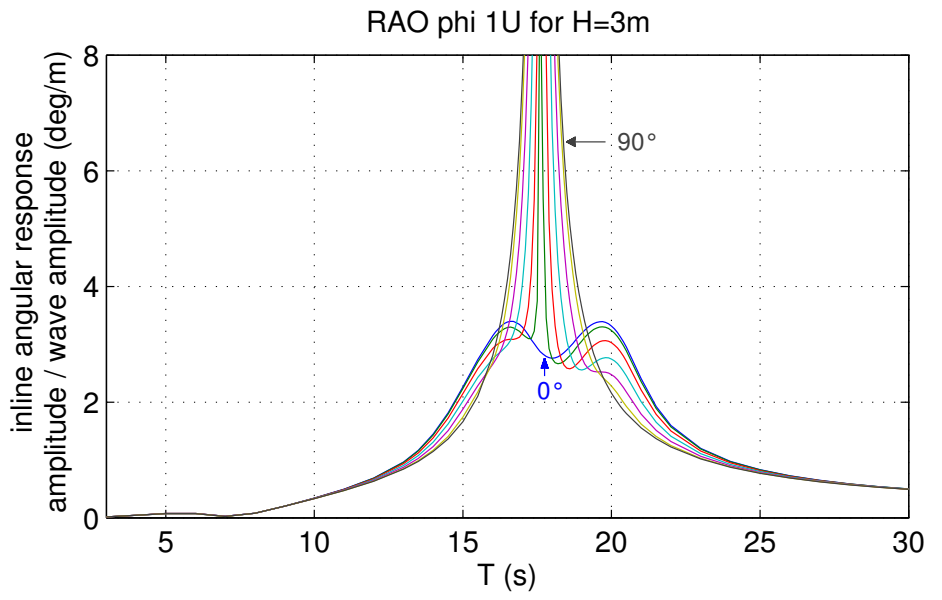

Figure 1: RAO of the float damped by a single TLCD for different incident angles

\begin{tabular}{|c|c|}
\hline Diameter, Height & $36 \mathrm{~m}, 9.5 \mathrm{~m}$ \\
\hline Draft, Freeboard & $5 \mathrm{~m}, 4.5 \mathrm{~m}$ \\
\hline Water Displacement & $5,089 \mathrm{~m}^{3}$ \\
\hline Mass, Including Ballast & $4,519,150 \mathrm{~kg}$ \\
\hline CM Location below SWL & $3.88238 \mathrm{~m}$ \\
\hline Roll Inertia about CM & $390,147,000 \mathrm{~kg} \cdot \mathrm{m}^{2}$ \\
\hline Pitch Inertia about CM & $390,147,000 \mathrm{~kg} \cdot \mathrm{m}^{2}$ \\
\hline Yaw Inertia about CM & $750,866,000 \mathrm{~kg} \cdot \mathrm{m}^{2}$ \\
\hline Anchor (Water) Depth & $200 \mathrm{~m}$ \\
\hline Separation between Opposing Anchors & $436 \mathrm{~m}$ \\
\hline Unstretched Line Length & $279.3 \mathrm{~m}$ \\
\hline Neutral Line Length Resting on Seabed & $0 \mathrm{~m}$ \\
\hline Line Diameter & $0.127 \mathrm{~m}$ \\
\hline Line Mass Density & $116 \mathrm{~kg} / \mathrm{m}$ \\
\hline Line Extensional Stiffness & $1,500,000,000 \mathrm{~N}$ \\
\hline
\end{tabular}

Table 2: Summary of MIT / NREL Barge Properties, from [10] 


\begin{tabular}{|c|c|}
\hline Rating & $5 \mathrm{MW}$ \\
\hline Rotor Orientation, Configuration & Upwind, 3 Blades \\
\hline Control Variable Speed & Collective Pitch \\
\hline Drivetrain High Speed & Multiple-Stage Gearbox \\
\hline Rotor, Hub Diameter & $126 \mathrm{~m}, 3 \mathrm{~m}$ \\
\hline Hub Height & $90 \mathrm{~m}$ \\
\hline Cut-In, Rated, Cut-Out Wind Speed & $3 \mathrm{~m} / \mathrm{s}, 11.4 \mathrm{~m} / \mathrm{s}, 25 \mathrm{~m} / \mathrm{s}$ \\
\hline Cut-In, Rated Rotor Speed & $6.9 \mathrm{rpm}, 12.1 \mathrm{rpm}$ \\
\hline Rated Tip Speed & $80 \mathrm{~m} / \mathrm{s}$ \\
\hline Overhang, Shaft Tilt, Precone & $5 \mathrm{~m}, 5^{\circ}, 2.5^{\circ}$ \\
\hline Rotor Mass & $110,000 \mathrm{~kg}$ \\
\hline Nacelle Mass & $240,000 \mathrm{~kg}$ \\
\hline Tower Mass & $347,460 \mathrm{~kg}$ \\
\hline Coordinate Location of Overall CM & $(-0.2 \mathrm{~m}, 0.0 \mathrm{~m}, 64.0 \mathrm{~m})$ \\
\hline
\end{tabular}

Table 3: Gross Properties Chosen for the NREL 5-MW Baseline Wind Turbine, from [10]

between the rotor and the wind because the damping induced is dependent on the controller chosen for the wind turbine (its impact can be negative or positive [13]). An illustration of the float with a 3S TLMCD is given in Figure 2.

\subsection{Assumptions}

To model the dynamics of the tank, we make the following assumptions:

1. the float is rigid. Therefore,

2. its centre of gravity, CoG, is immobile in the frame fixed to the barge,

3. the liquid in the TLCD is incompressible,

4. the column width is small with respect to length,

5. the flow of liquid in the tank is uniform in each column,

6. the position of the free surface of liquid in the tank is within the vertical column (i.e. vertical columns are never empty).

\subsection{Kinematics of the tank}

A TLCD is composed of two vertical tanks of cross-section $A_{v}$ connected by a horizontal duct of cross-section $A_{h}$. Liquid flows from one vertical column to the other through the horizontal tube. The restriction causing the damping (head loss) is located in the middle of the horizontal part. Fig. 3 is an illustration of the TLCD with the parameters presented in this subsection.

As we neglect the width of the columns, the TLCD geometry is defined by a line whose coordinates are expressed in the frame fixed to the barge

$$
r^{b}(\sigma) \triangleq\left[x_{t}^{b}, y_{t}^{b}(\sigma), z_{t}^{b}(\sigma)\right]^{\top}
$$

with

$$
y_{t}^{b}(\sigma) \triangleq\left\{\begin{array} { l l } 
{ \frac { L _ { h } } { 2 } } & { \sigma \leq - \frac { L _ { h } } { 2 } } \\
{ - \sigma } & { - \frac { L _ { h } } { 2 } < \sigma \leq \frac { L _ { h } } { 2 } } \\
{ - \frac { L _ { h } } { 2 } } & { \frac { L _ { h } } { 2 } < \sigma }
\end{array} \quad z _ { t } ^ { b } ( \sigma ) \triangleq \left\{\begin{array}{ll}
e+\frac{L_{h}}{2}+\sigma & \sigma \leq-\frac{L_{h}}{2} \\
e & -\frac{L_{h}}{2}<\sigma \leq \frac{L_{h}}{2} \\
e+\frac{L_{h}}{2}-\sigma & \frac{L_{h}}{2}<\sigma
\end{array}\right.\right.
$$




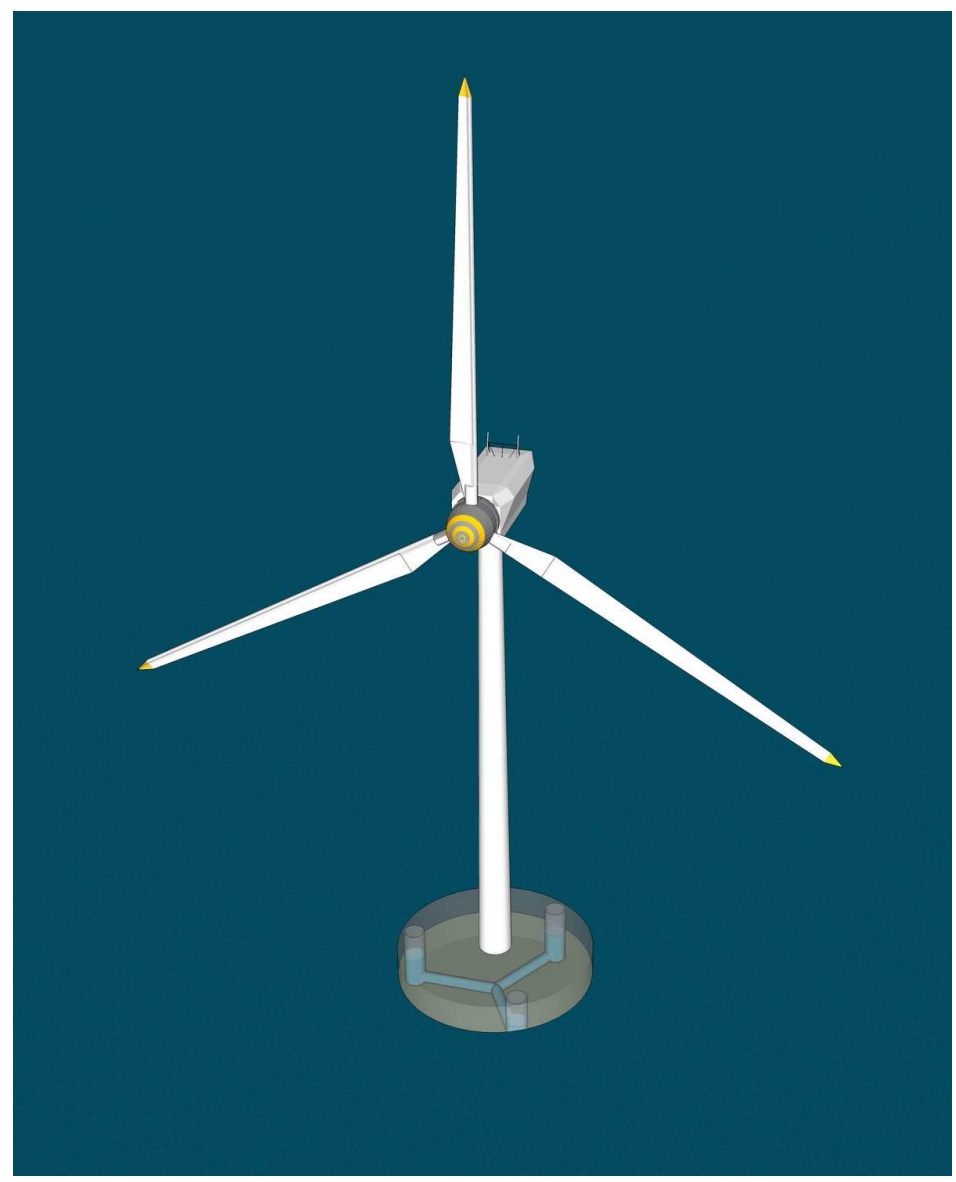

Figure 2: Illustration of the float with a 3S TLMCD

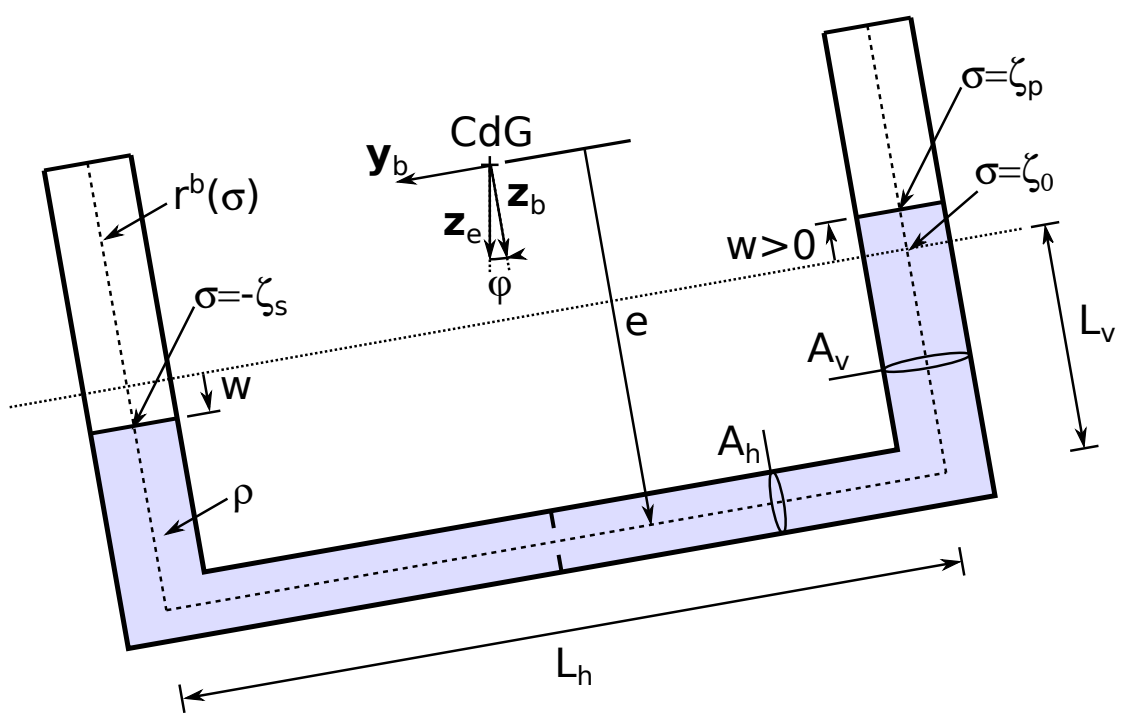

Figure 3: Scheme of a single TLCD illustrating the main variables 
where $x_{t}^{b}$ is defined for each damping system to generate a symmetric problem, and where $\sigma$ is the curvilinear abscissa along the geometry of the tank $(\sigma=0$ is at the centre of the horizontal tube, and for $\sigma>0 y_{t}^{b}(\sigma)$ is negative). We write $\frac{\mathrm{d} r^{b}}{\mathrm{~d} \sigma}(\sigma)$ as the unit vector tangent to the tank.

We define the cross-sectional area of the tank as

$$
A(\sigma) \triangleq \begin{cases}A_{v} & \sigma \leq-\frac{L_{h}}{2} \\ A_{h} & -\frac{L_{h}}{2}<\sigma \leq \frac{L_{h}}{2} \\ A_{v} & \frac{L_{h}}{2}<\sigma\end{cases}
$$

In this paper, the damping systems we consider consist of $N$ identical elementary subsystems (referred to as elements), which are regularly rotated around $\left(C o G, \mathbf{z}_{b}\right)$. The geometry of each element is given by $R_{z}\left(\alpha_{i}\right) r^{b}\left(\sigma_{i}\right)$ where

$$
R_{z}\left(\alpha_{i}\right)=\left[\begin{array}{ccc}
\cos \left(\alpha_{i}\right) & -\sin \left(\alpha_{i}\right) & 0 \\
\sin \left(\alpha_{i}\right) & \cos \left(\alpha_{i}\right) & 0 \\
0 & 0 & 1
\end{array}\right]
$$

is the rotation matrix around $\mathbf{z}$ and where $\alpha_{i}$ is the orientation angle of the $i^{t h}$ element. Let $v_{i}\left(\sigma_{i}\right)$ be the algebraic speed in in the $i^{\text {th }}$ element of the damping system. By convention, $v_{i}\left(\sigma_{i}\right)$ is positive if the liquid flows towards positive $\sigma$. The vector $\mathbf{v}_{i}^{b}\left(\sigma_{i}\right)$ is the speed of the liquid in the $i^{t h}$ element expressed in $\mathcal{R}_{b}$ as

$$
\mathbf{v}_{i}^{b}\left(\sigma_{i}\right)=v_{i}\left(\sigma_{i}\right) R_{z}\left(\alpha_{i}\right) \frac{\mathrm{d} \mathbf{r}^{b}}{\mathrm{~d} \sigma}\left(\sigma_{i}\right)
$$

We also introduce $\mathcal{V}_{h}$, the vector of algebraic speeds in the horizontal tubes, as

$$
\begin{gathered}
\mathcal{V}_{h} \triangleq\left[\begin{array}{c}
v_{1}(0) \\
\vdots \\
v_{N}(0)
\end{array}\right] \\
\mathcal{V}_{h}=P_{h} \dot{w}
\end{gathered}
$$

with $P_{h}$ given for each damping system in the Appendices.

\section{Linearised dynamics}

We define $X \triangleq\left[\begin{array}{llllll}\mathbf{x}^{n} & \Theta & w & \dot{\mathbf{x}}^{n} & \dot{\Theta} & \dot{w}\end{array}\right]^{\top}$ the state vector of our system, with $x^{n}=[x, y, z]^{\top} \in \mathbb{R}^{3}$ the position of the systems centre of gravity, $\Theta=$ $[\varphi, \theta, \psi]^{\top} \in \mathbb{R}^{3}$ the orientation of the float, $w \in \mathbb{R}^{n c}$ and $n c$ the number of variables describing the speed of the liquid inside the TLCD ( $n c$ will be detailed in $\S 4$ for each variant). The linearised model writes

$$
\dot{X}=\mathcal{A}(\omega) X+\mathcal{B}(\omega)\left[\begin{array}{c}
F_{\text {hydro }}(\omega, H) \\
Q_{\text {res }}(\eta)
\end{array}\right]
$$

with

$$
\mathcal{A}(\omega)=\left[\begin{array}{cc}
0_{6+n c \times 6+n c} & \mathrm{I}_{6+n c \times 6+n c} \\
(M(0)+A(\omega))^{-1} K & (M(0)+A(\omega))^{-1}(C(0,0)+B(\omega))
\end{array}\right]
$$



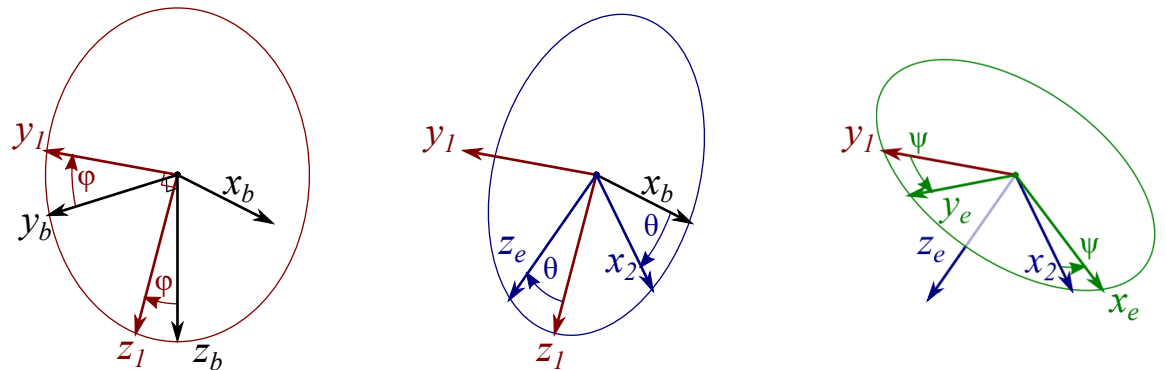

Figure 4: Orientation of $\mathcal{R}_{b}$ with respect to $\mathcal{R}_{e}$

$$
\mathcal{B}(\omega)=\left[\begin{array}{c}
0_{6+n c \times 6+n c} \\
(M(0)+A(\omega))^{-1}
\end{array}\right]
$$

where $M(0)$ and $C(0,0)$ the mass matrices given in $\S 4$ where $(q, \dot{q})=0$. The matrices $A(\omega)$ and $B(\omega)$ are respectively the radiation added mass and damping matrices, with $\omega$ the angular frequency of the monochromatic wave. The stiffness matrix $K$ accounts for buoyancy and gravity. The forces applied on the float and the liquid inside the TLCD are $F_{\text {hydro }}(\omega, H)$, depending on the angular frequency $\omega$ and $H$ the wave height, and $Q_{\text {res }}(\eta)$ as given in $\S 4.2$.

This linear model is based on the non-linear model presented in $\S 4$, which can be skipped by the reader, the system is tuned in $\S 5$, and the results of the numerical simulations are given in $\S 6$.

\section{Dynamic model of the damping systems}

\subsection{Description and properties of the frames}

In this paper, two frames are used: $\mathcal{R}_{b} \triangleq\left(C o G, \mathbf{x}_{b}, \mathbf{y}_{b}, \mathbf{z}_{b}\right)$ is the frame fixed to the barge, and $\mathcal{R}_{n} \triangleq\left(O, \mathbf{x}_{n}, \mathbf{y}_{n}, \mathbf{z}_{n}\right)$ is the Earth-fixed frame. Every vector $r \in \mathbb{R}^{3}$ is denoted by $r^{b}$ when expressed in the $b$ frame and $r^{n}$ in $\mathcal{R}_{n}$. The frames are oriented such as $\mathbf{z}$ points downwards.

The orientation of $\mathcal{R}_{b}$ with respect to $\mathcal{R}_{n}$ is defined by the "roll-pitch-yaw" Euler triple denoted by $\Theta=[\varphi, \theta, \psi]^{\top} \in \mathbb{R}^{3}$. The rotation matrix associated with $\Theta$ is

$$
R(\Theta) \triangleq\left[\begin{array}{ccc}
c_{\psi} c_{\theta} & -s_{\psi} c_{\varphi}+c_{\psi} s_{\theta} s_{\varphi} & s_{\psi} s_{\varphi}+c_{\psi} s_{\theta} c_{\varphi} \\
s_{\psi} c_{\theta} & c_{\psi} c_{\varphi}+s_{\psi} s_{\theta} s_{\varphi} & -c_{\psi} s_{\varphi}+s_{\psi} s_{\theta} c_{\varphi} \\
-s_{\theta} & c_{\theta} s_{\varphi} & c_{\theta} c_{\varphi}
\end{array}\right]
$$

with $c_{x}=\cos (x)$ and $s_{x}=\sin (x)$. Therefore, $\dot{\mathbf{x}}^{n}=R \mathbf{v}^{b}$, where $\mathbf{x}^{n}$ is the position of CoG in $\mathcal{R}_{n}$ expressed in the $n$ frame, and $\mathbf{v}^{b}$ the velocity of $\mathcal{R}_{b}$ relatively to $\mathcal{R}_{n}$ and expressed in $\mathcal{R}_{b}$. For all $\mathbf{u}=\left[u_{1}, u_{2}, u_{3}\right]^{\top} \in \mathbb{R}^{3}$, we define the cross-product matrix as

$$
S(\mathbf{u}) \triangleq\left[\begin{array}{ccc}
0 & -u_{3} & u_{2} \\
u_{3} & 0 & -u_{1} \\
-u_{2} & u_{1} & 0
\end{array}\right]=-S(\mathbf{u})^{\top}
$$


such that $\forall x, y \in \mathbb{R}^{3}, S(x) y=x \times y$. We denote by $\omega^{b}$ the rotation speed of the $b$ frame relative to the $n$ frame, expressed in $\mathcal{R}_{b}$. The time derivative of $R$ can then be given by [12]

$$
\dot{R}=R S\left(\omega^{b}\right)
$$

We define

$$
\begin{aligned}
G(\Theta) & \triangleq\left[\mathbf{x}, R\left([\varphi, 0,0]^{\top}\right)^{\top} \mathbf{y}, R(\Theta)^{\top} \mathbf{z}\right] \\
& =\left[\begin{array}{ccc}
1 & 0 & -s_{\theta} \\
0 & c_{\varphi} & s_{\varphi} c_{\theta} \\
0 & -s_{\varphi} & c_{\varphi} c_{\theta}
\end{array}\right]
\end{aligned}
$$

such that $\omega^{b}=G \dot{\Theta}$, with $\mathbf{x}, \mathbf{y}, \mathbf{z}$ as the unit vector along each axis.

We define $q \triangleq\left[\begin{array}{c}\mathbf{x}^{n} \\ \Theta \\ w\end{array}\right]$ and $v \triangleq\left[\begin{array}{c}\mathbf{v}^{b} \\ \omega^{b} \\ \dot{w}\end{array}\right]$, with $w \in \mathbb{R}^{n c}$ and $n c$ the number of variables describing the speed of the liquid inside the TLCD $(n c$ will be detailed in $\S 4$ for each variant). These variables are linked via $v=\mathcal{P} \dot{q}$ with

$$
\mathcal{P}(\Theta)=\left[\begin{array}{ccc}
R(\Theta)^{\top} & 0_{3 \times 3} & 0_{3 \times n c} \\
0_{3 \times 3} & G(\Theta) & 0_{3 \times n c} \\
0_{n c \times 3} & 0_{n c \times 3} & \mathbb{I}_{n c}
\end{array}\right]
$$

We have described the geometry and kinematics of the system, and now establish the dynamics of our systems using the Lagrangian approach. The dynamics of the system are classically given as

$$
\frac{\mathrm{d}}{\mathrm{d} t} \frac{\partial(T-V)}{\partial \dot{q}}-\frac{\partial(T-V)}{\partial q}=Q
$$

with $T$ the kinetic energy, $V$ the potential energy and $Q$ the generalized forces.

\subsection{Generalized forces}

To obtain $Q$ (the generalized forces), we express the power generated by external forces on our system as $\dot{q}^{\top} Q$. We write $Q \triangleq Q_{\text {hydro }}+Q_{\text {res }}$, with $Q_{\text {hydro }}$ the generalized force due to the interactions between the waves and the barge, and $Q_{r e s}$ the generalized force due to the restrictions in the TLMCD. For our simulations, the interactions between the platform and the water were modelled using a diffraction-radiation software. Following classical writing of the force generated by the fluid flow through the restriction, we write the forces $F_{h} \in \mathbb{R}^{N}$ in a damping system as

$$
F_{h}=-\frac{1}{2} \rho A_{h} \eta \circ \mathcal{V}_{h}(\dot{w}) \circ\left|\mathcal{V}_{h}(\dot{w})\right|
$$

with $\eta \in \mathbb{R}^{N}$ the vector of head-loss coefficients, $\rho$ the fluid density, and $\circ$ the Hadamard product (entrywise product). To establish the expression for $Q_{\text {res }}$, we express the power dissipated by the restrictions as $P_{\text {res }}=\mathcal{V}_{h}^{\top} F_{h}$, with $\mathcal{V}_{h}^{\top}=\dot{w}^{\top} P_{h}^{\top}$ according to (2). Therefore, $Q_{\text {res }}$ is given by

$$
Q_{\text {res }}(t, \dot{w})=\left[\begin{array}{c}
0_{6 \times 1} \\
P_{h}^{\top} F_{h}(\dot{w})
\end{array}\right]
$$




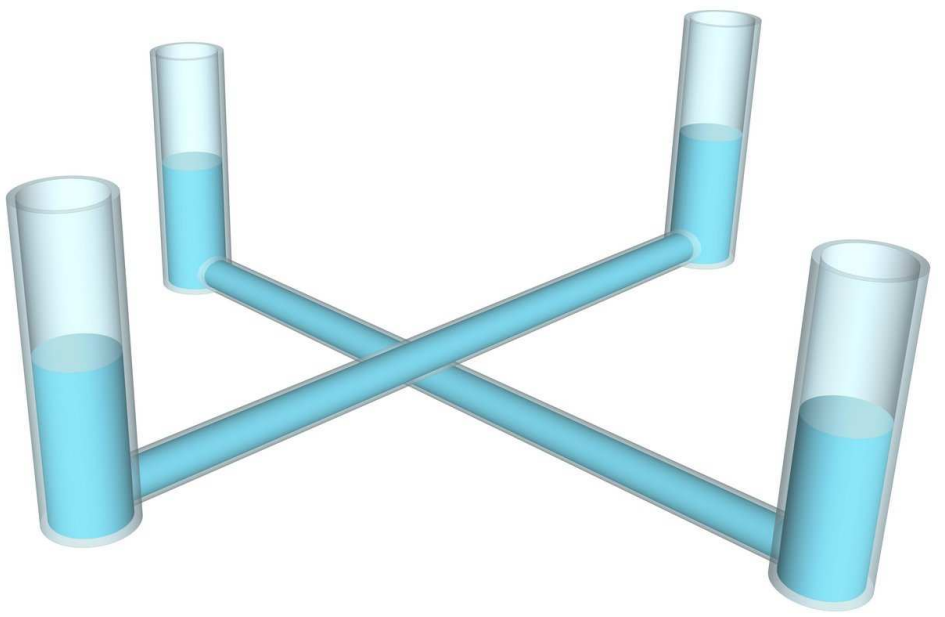

Figure 5: Geometry of the $2 \mathrm{U}$ damping system (the tubes do not intersect)

\subsection{System with $N$ TLCDs ( $N U)$}

We consider $N$ TLCDs regularly rotated around $\left(C o G, \mathbf{z}_{b}\right)$, and denote this system $N U$. As an example, the $2 \mathrm{U}$ system is illustrated in Fig. 5 . We set $x_{t}^{b}=0$ for our system to be axisymmetric. The orientation angle of each element writes $\alpha_{i}=\pi \frac{i-1}{N}$. To describe the position of the liquid, we need $N$ variables, i.e. $n c=N$. For the $N U$ system, each element is a TLCD, therefore, the curvilinear abscissa of each element, $\sigma_{i}$, ranges from $-\varsigma_{s i}$ to $\varsigma_{p i}$ defined as

$$
\begin{aligned}
& \varsigma_{p i}=\frac{L_{h}}{2}+L_{v}+w_{i} \\
& \varsigma_{s i}=\frac{L_{h}}{2}+L_{v}-w_{i}
\end{aligned}
$$

\subsubsection{Mechanical energy of the system}

The potential energy of the $N \mathrm{U}$ system is written as

$$
\begin{aligned}
V_{N U} & =\mathbf{z}^{\top} \cdot\left(g \rho \sum_{i=1}^{N} \int_{-\varsigma_{s i}}^{\varsigma_{p i}} A_{t}(\sigma)\left(R(\Theta) R_{z}\left(\alpha_{i}\right) \mathbf{r}^{b}(\sigma)+\mathbf{x}^{n}\right) \mathrm{d} \sigma\right) \\
& =-g m_{t} z-g \rho \mathbf{z}^{\top} R(\Theta)\left[\rho \sum_{i=1}^{N} \int_{-\varsigma_{s i}}^{\varsigma_{p i}} A_{t}(\sigma) R_{z}\left(\alpha_{i}\right) \mathbf{r}^{b}(\sigma) \mathrm{d} \sigma\right]
\end{aligned}
$$

where $g$ is the acceleration due to gravity, $m_{t}$ is the total mass of the liquid in the damping system.

The kinetic energy of the system is written as

$$
T_{N U}=\frac{1}{2} \dot{q}^{\top} \mathcal{M}_{N U}(q) \dot{q}
$$

with

$$
\mathcal{M}_{N U}(q) \triangleq \mathcal{P}(\Theta)^{\top} M_{N U}(w) \mathcal{P}(\Theta)=\mathcal{M}_{N U}^{\top} \in \mathbb{R}^{6+n c \times 6+n c}
$$

with $M_{N U}(w)$ as defined in (A.2). The calculation of the kinetic energy is detailed in Appendix A.1. 




Figure 6: The 3S TLMCD

\subsubsection{System dynamics}

We write the dynamics of the system as

$$
\mathcal{M}_{N U}(q) \ddot{q}+C_{N U}(q, \dot{q}) \dot{q}+k_{N U}(q)=Q_{h y d r o}+Q_{\text {res }_{N U}}(\dot{w})
$$

with $\mathcal{M}_{N U}(q)$ as defined in (7), and $C_{N U}, k_{N U}$ and $Q_{r_{e s} U}$ as defined in Appendix B.3.

\subsection{Model of a star-shaped TLMCD with $N$ elements (NS)}

This damping system is composed of $N$ halves of the TLCD interconnected at the coordinate $\mathbf{r}^{b}(\sigma=0)$ and regularly rotated around $\left(C o G, \mathbf{z}_{b}\right)$. We denote this system NS. For illustration purpose, the 3S system is shown in Fig. 6. For this system, each element is a half-TLCD, therefore the curvilinear abscissa of each element, $\sigma_{i}$, ranges from 0 to $\varsigma_{i}$. We still consider $x_{t}^{b}=0$. The orientation angle writes, $\alpha_{i}=\frac{2 \pi(i-1)}{N}$.

We note $\sigma=\varsigma_{i}$, the coordinate of the free surface of the $i^{t h}$ element. The total mass of the liquid is constant, and can be given by

$$
m_{t} \triangleq \rho \sum_{i=1}^{N} \int_{0}^{\varsigma_{i}} A_{t}\left(\sigma_{i}\right) \mathrm{d} \sigma_{i}
$$

If we know $\varsigma_{i}$ for $i=1, \ldots, N-1$, we can easily deduce $\varsigma_{N}$; therefore, $n c=N-1$. We define, for $i=1 . . n c$,

$$
\varsigma_{i}=\frac{L_{h}}{2}+L_{v}+w_{i}
$$

and

$$
\varsigma_{N}=\frac{L_{h}}{2}+L_{v}-\sum_{i=1}^{N-1} w_{i} .
$$

As shown in Appendix B.4, we write the dynamics of the system as

$$
\mathcal{M}_{N S}(q) \ddot{q}+C_{N S}(q, \dot{q}) \dot{q}+k_{N S}(q)=Q_{\text {hydro }}+Q_{\text {res }_{N S}}(\dot{w})
$$

where $\mathcal{M}_{N S}, C_{N S}, k_{N S}$ and $Q_{r e s_{N S}}$ are defined in Appendix B.4. 


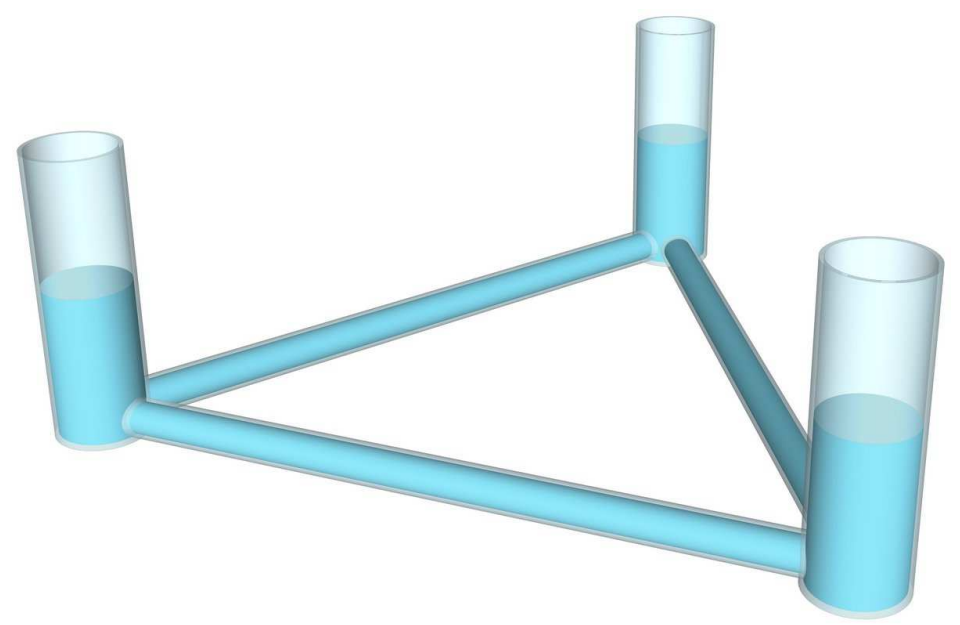

Figure 7: The 3P TLMCD

\subsection{Model of polygonal TLMCD with $N$ elements (NP)}

This damping system is composed of $N$ horizontal columns laid out to form a convex regular $N$-gon with $N$ vertical columns positioned at each intersection. We denote his system NP. The $3 \mathrm{P}$ case is shown in Fig. 7 . The elements of this system are composed of one horizontal tube and one vertical column, therefore, the curvilinear abscissa of each element, $\sigma_{i}$, ranges from $-\frac{L_{h}}{2}$ to $\varsigma_{i}$, as defined in (9). The geometry of our system implies $x_{t}^{b}=-\frac{L_{h}}{2 \tan \frac{\pi}{N}}$. The orientation angle $\alpha_{i}$ writes $\alpha_{i}=\frac{2 \pi(i-1)}{N}$, as in the NS problem.

There are $2 N$ values of the speed of the liquid (one for each horizontal tube and each vertical column). We can write $N$ local relations of flow conservation (at the base of each vertical column). We need $n c=2 N-N=N$ independent variables to know the speed of the liquid in each column. As the total mass of the liquid is constant, there are $N-1$ independent positions of free surfaces; therefore, we need to introduce an additional variable to completely describe the system. We arbitrarily choose $w_{n c}$ to be the "position" of the liquid in the $N^{t h}$ horizontal column.

The system's equations of motion are written as

$$
\mathcal{M}_{N P}(q) \ddot{q}+C_{N P}(q, \dot{q}) \dot{q}+k_{N P}(q)=Q_{h y d r o}+Q_{\text {res }_{N P}}(\dot{w})
$$

where $\mathcal{M}_{N P}, C_{N P}, k_{N P}$ and $Q_{r e s_{N P}}$ are defined in Appendix B.5.

\subsection{Results frame}

As we change the incidence of the waves, we need to change the results variables: we introduce $\varphi_{r}$ the inline angular response and $\theta_{r}$ the transverse angular response to describe the oscillations of the FWT along the direction of the waves and perpendicular to the waves, respectively. We need to express $\varphi_{r}$ and $\theta_{r}$ in terms of $\varphi, \theta$ and $\psi$. For this purpose, we introduced $\mathcal{R}_{e r}$ and $\mathcal{R}_{b r}$ as the "results frames". They are related via $R\left(\Theta_{r}\right)$ such that $\forall \mathbf{r} \in \mathbb{R}^{3}$,

$$
\mathbf{r}^{e r}=R\left(\Theta_{r}\right) \mathbf{r}^{b r}
$$


where $\Theta_{r} \triangleq\left[\varphi_{r}, \theta_{r}, \psi_{r}\right]^{\top}$. These frames are linked to $\mathcal{R}_{e}$ and $\mathcal{R}_{b}$ by a rotation around $\mathbf{z}$ at angle $\beta$, such that $\forall \mathbf{r} \in \mathbb{R}^{3}$,

$$
\mathbf{r}^{e}=R_{z}(\beta) \mathbf{r}^{e r}
$$

and

$$
\mathbf{r}^{b}=R_{z}(\beta) \mathbf{r}^{b r} .
$$

In $\S 4.1$ we defined $R(\Theta)$ so that

$$
\mathbf{r}^{e}=R(\Theta) \mathbf{r}^{b}
$$

thus, we write

$$
\mathbf{r}^{e r}=R_{z}^{\top}(\beta) \mathbf{r}^{e}=R_{z}^{\top}(\beta) R(\Theta) \mathbf{r}^{b}=R_{z}^{\top}(\beta) R(\Theta) R_{z}(\beta) \mathbf{r}^{b r},
$$

and by identification with (13), we get

$$
R\left(\Theta_{r}\right)=R_{z}^{\top}(\beta) R(\Theta) R_{z}(\beta) .
$$

Solving this equation yields $\Theta_{r}$ in terms of $\Theta$ and $\beta$.

\section{Tuning the proposed configurations}

Prior to assessing the robustness of each solution against wave incidence, we need to determine their design parameters. First, we must determine the mass of the liquid in the damper. We arbitrarily assume that each TLCD of the $2 \mathrm{U}$ variant weighs $2 \%$ of the total mass of the float, and that each TLMCD weighs $4 \%$ of the total mass, i.e. $2 \mathrm{U}, 3 \mathrm{~S}$ and $3 \mathrm{P}$ have the same mass. According to [27], the price of the system depends on three factors: the loss of space (occupied by the TLCD), additional construction costs, and the amount of steel needed for the tank. Since the space inside the barge has no commercial value, the cost of the loss of space is zero (if the system to damp was a building, the cost due to loss of space would have been the price of the floors occupied by the device). In our case, if the vertical columns were outside the float, additional construction costs would have incurred to ensure the structural integrity of the TLCD. To reduce this cost to zero, we designed the dampers to fit inside the barge.To determine the best design of each damper, we use the MATLAB fminsearch optimisation function, with the following performance index to be minimized:

$$
\text { P.I. }=\max _{T \in[3 ; 30]}(|\varphi|)
$$

where $|\varphi|$ is the steady state roll magnitude obtained via a simulation for each period of monochromatic wave (excitation). It is a min-max problem where the decision variables are $L_{h}, L_{v}, \nu$ and $\eta$. This problem is solved under constraints $L_{h} \leq L_{h_{\max i}}$ and $L_{v} \leq L_{v_{\operatorname{maxi}}}$ to fit the damper inside the barge so that the construction cost remains zero. To avoid a violation of assumption 6 , we set $L_{v}=L_{v_{\max i}}$.

In a previous paper [3], we considered damping with a single TLCD using the same float subject to waves in the vertical plan $x_{t}=0$. The results showed that the optimal value of $L_{h}$ was $L_{h_{\max i}}$. Therefore, we chose to set $L_{h}=L_{h_{\max i}}$ to reduce the number of variables in the optimization problem. As we have 


\begin{tabular}{|c|c|c||c|c|c||c|}
\cline { 2 - 7 } \multicolumn{1}{c|}{} & $\nu$ & $\eta$ & $L_{h}$ & $A_{v}$ & $\mu$ & $P . I$. \\
\hline 2U & 4.06 & 5.90 & $32.31 \mathrm{~m}$ & $5.68 \mathrm{~m}^{2}$ & $4 \%$ & 3.50 \\
\hline 3S & 4.12 & 3.43 & $31.88 \mathrm{~m}$ & $7.67 \mathrm{~m}^{2}$ & $4 \%$ & 3.57 \\
\hline 3P & 7.11 & 10.72 & $27.61 \mathrm{~m}$ & $7.65 \mathrm{~m}^{2}$ & $4 \%$ & 3.54 \\
\hline
\end{tabular}

Table 4: Optimal TLMCD parameters for a wave height $H=3 \mathrm{~m}$

$L_{h}=L_{h_{\max i}}$ and $L_{v}=L_{v_{\max i}}$, the position of the TLMCD inside the barge is imposed.

We define $\mu \triangleq \frac{m_{t}}{M_{S_{11}}}$ as the ratio of the mass of the liquid in the TLMCD to the total mass of the float. We summarize the design of each damper in Table 4 for a given wave height $H=3 \mathrm{~m}$ and an incidence of $\beta=0^{\circ}$. As the natural period of the float is close to the predominant period of extreme sea states (15 s-20 s), we chose the performance index to damp this resonance. Note that for a given site, we could have used an adapted performance index to obtain the design best suited to the conditions of the local sea.

We also note that $\nu$ (the cross-section ratio) of the $3 \mathrm{P}$ system is much larger, which means that the $3 \mathrm{P}$ system has a lower resonant period for the same $\nu$.

\section{Simulation results}

In the previous section we detailed the design of each damping system. In this section we perform numerical simulations to compare their robustness against wave incidence. As the dampers are tuned to the roll/pitch natural frequency, they have almost no effect on the other motions of the wind turbine. This is why in this section we only deal with the roll and pitch motions.

\subsection{Preliminary considerations}

Before we perform numerical simulations, let's consider the following points.

Evaluation criterion: The RAO. We introduce the response amplitude operator $(\mathrm{RAO})$. It is defined as the ratio of the system's motion to the wave amplitude causing it, and is represented over a range of (monochromatic) wave periods [9]. It is employed as a quantitative evaluation tool for the rest of the study.

Results frames We remind the reader that we introduced the results frame in $\S 4.6$. As we use the linear model, the states of the results frame are linked to the original states via $\mathbf{x}^{n r}=R_{z}^{\top}(\beta) \mathbf{x}^{n}$ and $\Theta^{r}=R_{z}^{\top}(\beta) \Theta$.

\subsection{Numerical simulations}

We simulated the system's response to a $3 \mathrm{~m}$ wave excitation until a steady state was attained. We plotted the RAOs for monochromatic waves of periods ranging from $3 \mathrm{~s}$ to $30 \mathrm{~s}$ as well as for different incident angles. It has been verified that the vertical columns were never empty during the simulations.

We plotted the results in Fig. 8. Due to the symmetries of the damping systems, we plotted curves between $0^{\circ}$ and $45^{\circ}$ for the $2 \mathrm{U}$ case, and between $0^{\circ}$ and $30^{\circ}$ for the $3 \mathrm{~S}$ and $3 \mathrm{P}$ cases.

In Fig. 8, we can see that the $3 \mathrm{~S}$ and $3 \mathrm{P}$ systems are more robust against wave incidence than the $2 \mathrm{U}$ damper. All dampers create a parasitic transverse 

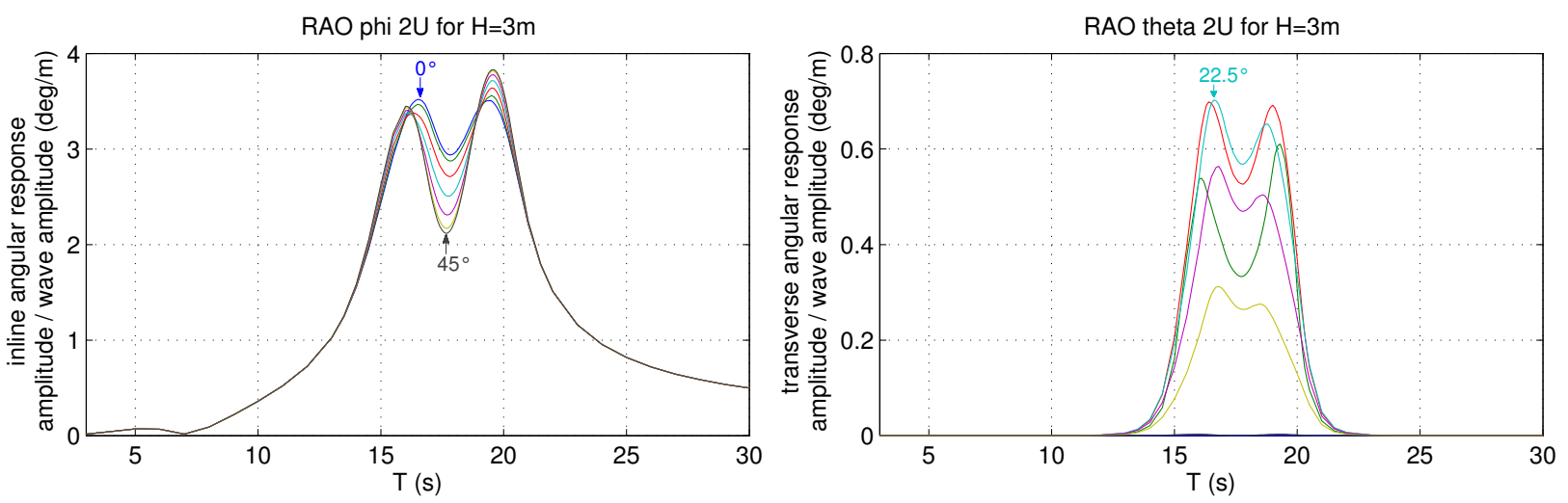

(a) RAO of the $2 \mathrm{U}$ system
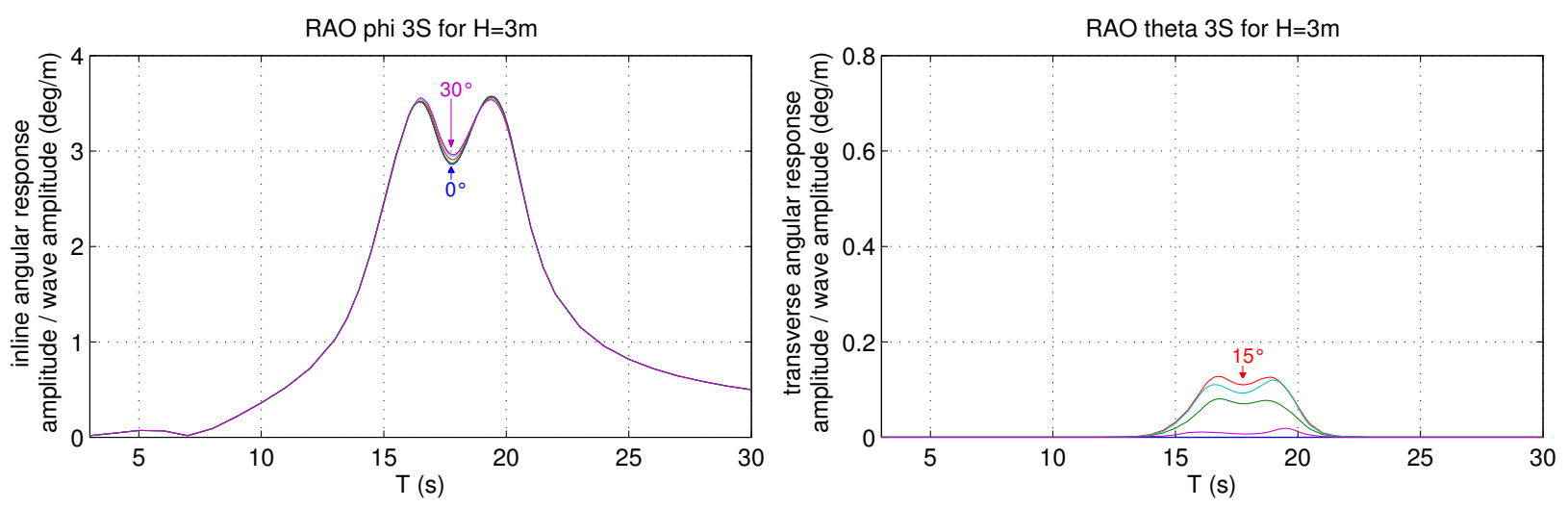

(b) RAO of the $3 \mathrm{~S}$ system


(c) RAO of the $3 \mathrm{P}$ system

Figure 8: RAO of an arrangement of multiple TLCDs (a) and variants of TLMCDs ((b) and (c)) for different wave incidences of a $3 \mathrm{~m}$ monochromatic wave 
angular response, but it is worth noting that the $2 \mathrm{U}$ system creates significantly greater parasitic transverse angular motion than the $3 \mathrm{P}$ and $3 \mathrm{~S}$ dampers.

\section{Conclusions}

In this paper, we introduced the concept of a tuned liquid multi-column damper to damp systems with similar pitch and roll behaviours, e.g. an offshore platform. This damper was inspired by the tuned liquid column damper (TLCD). We developed dynamic models of an offshore platform coupled with different variants of TLMCDs and compared them against a reference system consisting of an arrangement of multiple TLCDs. The results of simulations showed that the two proposed systems (3S and $3 \mathrm{P}$ ) are more robust against variations in wave incidence than a crosswise layout of two TLCDs.

In this study, all considered devices are passive. However further work will focus on the semi-active control of these devices, i.e. changing the head loss coefficients $\eta$ continually to achieve better performance.

\section{References}

[1] C. C. Chang and C. T. Hsu. Control performance of liquid column vibration absorbers. Engineering Structures, 20(7):580-586, 1998 . doi: 10.1016/S0141-0296(97)00062-X.

[2] S. Christiansen, S. M. Tabatabaeipour, T. Bak, and T. Knudsen. Wave disturbance reduction of a floating wind turbine using a reference modelbased predictive control. In American Control Conference (ACC), 2013, pages 2214-2219. IEEE, 2013. doi: 10.1109/ACC.2013.6580164.

[3] C. Coudurier, O. Lepreux, and N. Petit. Passive and semi-active control of an offshore floating wind turbine using a tuned liquid column damper. IFAC-PapersOnLine, 48(16):241-247, 2015. doi: 10.1016/j.ifacol.2015.10.287.

[4] A. Di Matteo, F. Lo Iacono, G. Navarra, and A. Pirrotta. Direct evaluation of the equivalent linear damping for TLCD systems in random vibration for pre-design purposes. International Journal of Non-Linear Mechanics, 63:19 - 30, 2014. ISSN 0020-7462. doi: 10.1016/j.ijnonlinmec.2014.03.009.

[5] Ernst \& Young. Offshore wind in europe: Walking the tightrope to success. Technical report, European Wind Energy Association, 2015.

[6] H. Frahm. Results of trials of the anti-rolling tanks at sea. Journal of the American Society for Naval Engineers, 23(2):571-597, 1911. doi: 10.1111/j.1559-3584.1911.tb04595.x.

[7] H. Gao, K. Kwok, and B. Samali. Optimization of tuned liquid column dampers. Engineering Structures, 19(6):476 - 486, 1997. ISSN 0141-0296. doi: 10.1016/S0141-0296(96)00099-5.

[8] C. Holden and T. I. Fossen. A nonlinear 7-dof model for Utanks of arbitrary shape. Ocean Engineering, 45:22-37, 2012. doi: 10.1016/j.oceaneng.2012.02.002. 
[9] International Organization for Standardization. Iso 13624-1-2009 petroleum and natural gas industries: Drilling and production equipment - part 1: Design and operation of marine drilling riser equipment, 2009.

[10] J. M. Jonkman. Dynamics modeling and loads analysis of an offshore floating wind turbine. $\mathrm{PhD}$ thesis, National Renewable Energy Laboratory, 2007.

[11] M. A. Lackner and M. A. Rotea. Structural control of floating wind turbines. Mechatronics, 21(4):704-719, 2011. doi: 10.1016/j.mechatronics.2010.11.007.

[12] L. Landau and E. Lifshitz. Mechanics. Elsevier, third edition, 1976.

[13] T. J. Larsen and T. D. Hanson. A method to avoid negative damped low frequent tower vibrations for a floating, pitch controlled wind turbine. In Journal of Physics: Conference Series, volume 75. IOP Publishing, 2007. doi: 10.1088/1742-6596/75/1/012073.

[14] N. Luo, C. L. Bottasso, H. R. Karimi, and M. Zapateiro. Semiactive control for floating offshore wind turbines subject to aero-hydro dynamic loads. International Conference on Renewable Energies and Power Quality, 2011.

[15] R. Moaleji and A. R. Greig. On the development of ship anti-roll tanks. Ocean Engineering, 34(1):103 - 121, 2007. ISSN 0029-8018. doi: 10.1016/j.oceaneng.2005.12.013.

[16] W. Musial, S. Butterfield, B. Ram, et al. Energy from offshore wind. In Offshore technology conference, pages 1888-1898. Offshore Technology Conference, 2006.

[17] H. Namik. Individual blade pitch and disturbance accommodating control of floating offshore wind turbines. PhD thesis, ResearchSpace Auckland, 2012.

[18] H. Namik, M. Rotea, and M. Lackner. Active structural control with actuator dynamics on a floating wind turbine. In 51st AIAA Aerospace Sciences Meeting including the New Horizons Forum and Aerospace Exposition, 2013. doi: 10.2514/6.2013-455.

[19] National Renewable Energy Laboratory. Renewable Energy Data Book. U.S. Department of Energy, 2012.

[20] T. Perez and M. Blanke. Ship roll damping control. Annual Reviews in Control, 36(1):129-147, 2012. doi: 10.1016/j.arcontrol.2012.03.010.

[21] T. E. Saeed, G. Nikolakopoulos, J.-E. Jonasson, and H. Hedlund. A stateof-the-art review of structural control systems. Journal of Vibration and Control, 2013. doi: 10.1177/1077546313478294.

[22] M. Shadman and A. Akbarpour. Utilizing TLCD (tuned liquid column damper) in floating wind turbines. In ASME 2012 31st International Conference on Ocean, Offshore and Arctic Engineering, pages 241-247. American Society of Mechanical Engineers, 2012. doi: 10.1115/OMAE201283330 . 
[23] Y. Si, H. R. Karimi, and H. Gao. Modelling and optimization of a passive structural control design for a spar-type floating wind turbine. Engineering Structures, 69(0):168 - 182, 2014. ISSN 0141-0296. doi: 10.1016/j.engstruct.2014.03.011.

[24] G. Stewart and M. Lackner. Offshore wind turbine load reduction employing optimal passive tuned mass damping systems. IEEE transactions on control systems technology, 21(4):1090 - 1104, 2013. doi: 10.1109/TCST.2013.2260825.

[25] J.-C. Wu, C.-H. Chang, and Y.-Y. Lin. Optimal designs for nonuniform tuned liquid column dampers in horizontal motion. Journal of Sound and Vibration, 326(1-2):104 - 122, 2009. ISSN 0022-460X. doi: 10.1016/j.jsv.2009.04.027.

[26] S. Yalla and A. Kareem. Optimum absorber parameters for tuned liquid column dampers. Journal of Structural Engineering, 126(8):906-915, 2000. doi: 10.1061/(ASCE)0733-9445(2000)126:8(906).

[27] S. K. Yalla. Liquid dampers for mitigation of structural response: theoretical development and experimental validation. $\mathrm{PhD}$ thesis, University of Notre Dame, 2001. 


\section{Appendix A. Kinetic and potential energy of the proposed dampers}

Appendix A.1. Kinetic energy of the $N U$ damper

Following the method used in [8, Appendix B], we compute the kinetic energy of the $N U$ system.

For the $N \mathrm{U}$ system and for $i=1, \ldots, N$

$$
v_{i}\left(\sigma_{i}\right)=\frac{A_{v}}{A_{t}\left(\sigma_{i}\right)} \dot{w}_{i}
$$

we write $\mathbf{v}_{i}^{b}\left(\sigma_{i}\right)$ according to (1)

$$
\mathbf{v}_{i}^{b}\left(\sigma_{i}\right)=\frac{A_{v}}{A_{t}\left(\sigma_{i}\right)} \dot{w}_{i} R_{z}\left(\alpha_{i}\right) \frac{\mathrm{d} \mathbf{r}^{b}}{\mathrm{~d} \sigma}\left(\sigma_{i}\right) .
$$

Therefore, matrix $P_{h_{N U}}$ appearing in (2) can be given by

$$
P_{h_{N U}}=\nu \mathbb{I}_{n c}
$$

We write the kinetic energy of the system as

$$
T_{N U}=T_{s}+T_{D N U}
$$

where

$$
T_{s}=\frac{1}{2} v^{\top}\left[\begin{array}{cc}
M_{s} & 0_{6 \times n c} \\
0_{n c \times 6} & 0_{n c \times n c}
\end{array}\right] v
$$

with $M_{s}$ the float mass matrix, and

$$
\begin{aligned}
T_{D N U} & =\frac{1}{2} \rho \sum_{i=1}^{N} \int_{-\varsigma_{s i}}^{\varsigma_{p i}} A_{t}\left(\sigma_{i}\right)\left\|\mathbf{v}^{b}+\omega^{b} \times R_{z}\left(\alpha_{i}\right) \mathbf{r}^{b}\left(\sigma_{i}\right)+\mathbf{v}_{i}^{b}\left(\sigma_{i}\right)\right\|^{2} \mathrm{~d} \sigma_{i} \\
& =\frac{1}{2} \sum_{i=1}^{N}\left(\rho \int_{-\varsigma_{s i}}^{\varsigma_{p i}} A_{t}\left(\sigma_{i}\right) \mathrm{d} \sigma_{i}\right)\left\|\mathbf{v}^{b}\right\|^{2}-\frac{1}{2} \omega^{b \top}\left(\rho \sum_{i=1}^{N} \int_{-\varsigma_{s i}}^{\varsigma_{p i}} A_{t}\left(\sigma_{i}\right) S^{2}\left(R_{z}\left(\alpha_{i}\right) \mathbf{r}^{b}\left(\sigma_{i}\right)\right) \mathrm{d} \sigma_{i}\right) \omega^{b} \\
& +\omega^{b \top}\left(\rho \sum_{i=1}^{N} \int_{-\varsigma_{s i}}^{\varsigma_{p i}} A_{t}\left(\sigma_{i}\right) S\left(R_{z}\left(\alpha_{i}\right) \mathbf{r}^{b}\left(\sigma_{i}\right)\right) \mathrm{d} \sigma_{i}\right) \mathbf{v}^{b}+\mathbf{v}^{b \top}\left(\rho A_{v} \sum_{i=1}^{N} \int_{-\varsigma_{s i}}^{\varsigma_{p i}} R_{z}\left(\alpha_{i}\right) \frac{\mathrm{d} \mathbf{r}^{b}}{\mathrm{~d} \sigma}\left(\sigma_{i}\right) \dot{w}_{i} \mathrm{~d} \sigma_{i}\right) \\
& +\omega^{b \top}\left(\rho A_{v} \sum_{i=1}^{N} \int_{-\varsigma_{s i}}^{\varsigma_{p i}} S\left(R_{z}\left(\alpha_{i}\right) \mathbf{r}^{b}\left(\sigma_{i}\right)\right) R_{z}\left(\alpha_{i}\right) \frac{\mathrm{d} \mathbf{r}^{b}}{\mathrm{~d} \sigma}\left(\sigma_{i}\right) \dot{w}_{i} \mathrm{~d} \sigma_{i}\right) \\
& +\frac{1}{2} \sum_{i=1}^{N}\left(\rho A_{v}^{2} \int_{-\varsigma_{s i}}^{\varsigma_{p i}} \frac{\dot{w}_{i}^{2}}{A_{t}\left(\sigma_{i}\right)} \mathrm{d} \sigma_{i}\right) .
\end{aligned}
$$

Therefore, we can write

$$
T_{N U}=\frac{1}{2} v^{\top} M_{N U}(w) v=\frac{1}{2} \dot{q}^{\top} \mathcal{M}_{N U}(q) \dot{q},
$$

with $\mathcal{M}_{N U} \triangleq \mathcal{P}^{\top} M_{N U} \mathcal{P}$ and

$$
M_{N U}(w) \triangleq\left[\begin{array}{cc}
M_{s} & 0_{6 \times n c} \\
0_{n c \times 6} & 0_{n c \times n c}
\end{array}\right]+\left[\begin{array}{ccc}
m_{t} \mathbb{I}_{3} & M_{v \omega}(w) & M_{v q}(w) \\
M_{v \omega}^{\top}(w) & M_{\omega}(w) & M_{\omega q}(w) \\
M_{v q}^{\top}(w) & M_{\omega q}^{\top}(w) & M_{q}(w)
\end{array}\right] .
$$


For $i=1, \ldots, n c$,

$$
\begin{gathered}
m_{t} \triangleq \rho \sum_{i=1}^{N} \int_{-\varsigma_{s i}}^{\varsigma_{p i}} A_{t}\left(\sigma_{i}\right) \mathrm{d} \sigma_{i} \in \mathbb{R} \\
M_{v \omega} \triangleq-\rho \sum_{i=1}^{N} \int_{-\varsigma_{s i}}^{\varsigma_{p i}} A_{t}\left(\sigma_{i}\right) S\left(R_{z}\left(\alpha_{i}\right) \mathbf{r}^{b}\left(\sigma_{i}\right)\right) \mathrm{d} \sigma_{i}=-M_{v \omega}^{\top}(w) \in \mathbb{R}^{3 \times 3} \\
M_{\omega} \triangleq-\rho \sum_{i=1}^{N} \int_{-\varsigma_{s i}}^{\varsigma_{p i}} A_{t}\left(\sigma_{i}\right) S^{2}\left(R_{z}\left(\alpha_{i}\right) \mathbf{r}^{b}\left(\sigma_{i}\right)\right) \mathrm{d} \sigma_{i}=M_{\omega}^{\top}(w) \in \mathbb{R}^{3 \times 3} \\
M_{v q}[:, i] \triangleq \rho A_{v} \int_{-\varsigma_{s i}}^{\varsigma_{p i}} R_{z}\left(\alpha_{i}\right) \frac{\mathrm{d} \mathbf{r}^{b}}{\mathrm{~d} \sigma}\left(\sigma_{i}\right) \mathrm{d} \sigma_{i} \in \mathbb{R}^{3 \times 1} \\
M_{\omega q}[:, i] \triangleq \rho A_{v} \int_{-\varsigma_{s i}}^{\varsigma_{p i}} S\left(R_{z}\left(\alpha_{i}\right) \mathbf{r}^{b}\left(\sigma_{i}\right)\right) R_{z}\left(\alpha_{i}\right) \frac{\mathrm{d} \mathbf{r}^{b}}{\mathrm{~d} \sigma}\left(\sigma_{i}\right) \mathrm{d} \sigma \in \mathbb{R}^{3 \times 1} \\
M_{q} \triangleq \mathbb{I}_{n c} \rho A_{v}\left(L_{h} \nu+2 L_{v}\right) \in \mathbb{R}^{n c}
\end{gathered}
$$

with $M_{v q} \in \mathbb{R}^{3 \times n c}, M_{\omega q} \in \mathbb{R}^{3 \times n c}$.

Appendix A.2. Kinetic and potential energy of the $N S$ damper

Following the method used in Appendix A.1 for the NS variant, we write

$$
M_{N S}(w) \triangleq\left[\begin{array}{cc}
M_{s} & 0_{6 \times n c} \\
0_{n c \times 6} & 0_{n c \times n c}
\end{array}\right]+\left[\begin{array}{ccc}
m_{t} \mathbb{I}_{3} & M_{v \omega}(w) & M_{v q}(w) \\
M_{v \omega}^{\top}(w) & M_{\omega}(w) & M_{\omega q}(w) \\
M_{v q}^{\top}(w) & M_{\omega q}^{\top}(w) & M_{q}(w)
\end{array}\right]
$$

with, for $j=1, \ldots, n c$,

$$
\begin{aligned}
& m_{t} \triangleq \rho \sum_{i=1}^{N} \int_{0}^{\varsigma_{i}} A_{t}\left(\sigma_{i}\right) \mathrm{d} \sigma_{i} \in \mathbb{R} \\
& M_{v \omega} \triangleq-\rho \sum_{i=1}^{N} \int_{0}^{\varsigma_{i}} A_{t}\left(\sigma_{i}\right) S\left(R_{z}\left(\alpha_{i}\right) \mathbf{r}^{b}\left(\sigma_{i}\right)\right) \mathrm{d} \sigma_{i}=-M_{v \omega}^{\top}(w) \in \mathbb{R}^{3 \times 3} \\
& M_{\omega} \triangleq-\rho \sum_{i=1}^{N} \int_{0}^{\varsigma_{i}} A_{t}\left(\sigma_{i}\right) S^{2}\left(R_{z}\left(\alpha_{i}\right) \mathbf{r}^{b}\left(\sigma_{i}\right)\right) \mathrm{d} \sigma_{i}=M_{\omega}^{\top}(w) \in \mathbb{R}^{3 \times 3} \\
& M_{v q}[:, j] \triangleq \rho A_{v} P_{h_{N S}}[:, j] \sum_{i=1}^{N} \int_{0}^{\varsigma_{i}} R_{z}\left(\alpha_{i}\right) \frac{\mathrm{d} \mathbf{r}^{b}}{\mathrm{~d} \sigma}\left(\sigma_{i}\right) \mathrm{d} \sigma_{i} \in \mathbb{R}^{3 \times 1} \\
& M_{\omega q}[:, j] \triangleq \rho A_{v} P_{h_{N S}}[:, j] \sum_{i=1}^{N} \int_{0}^{\varsigma_{i}} S\left(R_{z}\left(\alpha_{i}\right) \mathbf{r}^{b}\left(\sigma_{i}\right)\right) R_{z}\left(\alpha_{i}\right) \frac{\mathrm{d} \mathbf{r}^{b}}{\mathrm{~d} \sigma}\left(\sigma_{i}\right) \mathrm{d} \sigma \in \mathbb{R}^{3 \times 1} \\
& M_{q} \triangleq \rho A_{v}\left(P_{h_{N S}}^{\top} \frac{L_{h}}{2 \nu}(\nu-1)+\nu^{-2} P_{h_{N S}}^{\top}\left[\begin{array}{ccccc}
\varsigma_{1} & 0 & \cdots & \cdots & 0 \\
0 & \ddots & \ddots & & \vdots \\
\vdots & \ddots & \ddots & \ddots & \vdots \\
\vdots & & \ddots & \ddots & 0 \\
0 & \cdots & \cdots & 0 & \varsigma_{N}
\end{array}\right] P_{h_{N S}}\right) \in \mathbb{R}^{n c}
\end{aligned}
$$


with $M_{v q} \in \mathbb{R}^{3 \times n c}, M_{\omega q} \in \mathbb{R}^{3 \times n c}$, and

$$
P_{h_{N S}} \triangleq \nu\left[\begin{array}{c}
\mathbb{I}_{n c} \\
-\mathbb{1}_{1 \times n c}
\end{array}\right] .
$$

The potential energy of the NS system is written as

$$
\begin{aligned}
V_{N S} & =\mathbf{z}^{\top} \cdot\left(g \rho \sum_{i=1}^{N} \int_{0}^{\varsigma_{i}} A_{t}(\sigma)\left(R(\Theta) R_{z}\left(\alpha_{i}\right) \mathbf{r}^{b}(\sigma)+\mathbf{x}^{n}\right) \mathrm{d} \sigma\right) \\
& =-g m_{t} z-g \rho \mathbf{z}^{\top} R(\Theta)\left[\rho \sum_{i=1}^{N} \int_{0}^{\varsigma_{i}} A_{t}(\sigma) R_{z}\left(\alpha_{i}\right) \mathbf{r}^{b}(\sigma) \mathrm{d} \sigma\right]
\end{aligned}
$$

Appendix A.3. Kinetic and potential energy of the NP damper

Following the method used in Appendix A.1 for the NP variant, we write

$$
M_{N P}(w) \triangleq\left[\begin{array}{cc}
M_{s} & 0_{6 \times n c} \\
0_{n c \times 6} & 0_{n c \times n c}
\end{array}\right]+\left[\begin{array}{ccc}
m_{t} \mathbb{I}_{3} & M_{v \omega}(w) & M_{v q}(w) \\
M_{v \omega}^{\top}(w) & M_{\omega}(w) & M_{\omega q}(w) \\
M_{v q}^{\top}(w) & M_{\omega q}^{\top}(w) & M_{q}(w)
\end{array}\right]
$$

with, for $j=1, \ldots, n c$,

$$
\begin{aligned}
& m_{t} \triangleq \rho \sum_{i=1}^{N} \int_{-\frac{L_{h}}{2}}^{\varsigma_{i}} A_{t}\left(\sigma_{i}\right) \mathrm{d} \sigma_{i} \in \mathbb{R} \\
& M_{v \omega} \triangleq-\rho \sum_{i=1}^{N} \int_{-\frac{L_{h}}{2}}^{\varsigma_{i}} A_{t}\left(\sigma_{i}\right) S\left(R_{z}\left(\alpha_{i}\right) \mathbf{r}^{b}\left(\sigma_{i}\right)\right) \mathrm{d} \sigma_{i}=-M_{v \omega}^{\top}(w) \in \mathbb{R}^{3 \times 3} \\
& M_{\omega} \triangleq-\rho \sum_{i=1}^{N} \int_{-\frac{L_{h}}{2}}^{\varsigma_{i}} A_{t}\left(\sigma_{i}\right) S^{2}\left(R_{z}\left(\alpha_{i}\right) \mathbf{r}^{b}\left(\sigma_{i}\right)\right) \mathrm{d} \sigma_{i}=M_{\omega}^{\top}(w) \in \mathbb{R}^{3 \times 3} \\
& M_{v q}[:, j] \triangleq \rho A_{v} P_{h_{N P}}[:, j] \sum_{i=1}^{N} \int_{-\frac{L_{h}}{2}}^{\frac{L_{h}}{2}} R_{z}\left(\alpha_{i}\right) \frac{\mathrm{d} \mathbf{r}^{b}}{\mathrm{~d} \sigma}\left(\sigma_{i}\right) \mathrm{d} \sigma_{i} \\
& +\rho A_{v} P_{h 2_{N P}}[:, j] \sum_{i=1}^{N} \int_{\frac{L_{h}}{2}}^{\varsigma_{i}} R_{z}\left(\alpha_{i}\right) \frac{\mathrm{d} \mathbf{r}^{b}}{\mathrm{~d} \sigma}\left(\sigma_{i}\right) \mathrm{d} \sigma_{i} \in \mathbb{R}^{3 \times 1} \\
& M_{\omega q}[:, j] \triangleq \rho A_{v} P_{h_{N P}}[:, j] \sum_{i=1}^{N} \int_{-\frac{L_{h}}{2}}^{\frac{L_{h}}{2}} S\left(R_{z}\left(\alpha_{i}\right) \mathbf{r}^{b}\left(\sigma_{i}\right)\right) R_{z}\left(\alpha_{i}\right) \frac{\mathrm{d} \mathbf{r}^{b}}{\mathrm{~d} \sigma}\left(\sigma_{i}\right) \mathrm{d} \sigma \\
& +\rho A_{v} P_{h 2_{N P}}[:, j] \sum_{i=1}^{N} \int_{\frac{L_{h}}{2}}^{\varsigma_{i}} S\left(R_{z}\left(\alpha_{i}\right) \mathbf{r}^{b}\left(\sigma_{i}\right)\right) R_{z}\left(\alpha_{i}\right) \frac{\mathrm{d} \mathbf{r}^{b}}{\mathrm{~d} \sigma}\left(\sigma_{i}\right) \mathrm{d} \sigma \in \mathbb{R}^{3 \times 1} \\
& M_{q} \triangleq \rho A_{v}\left(\frac{L_{h}}{\nu} P_{h_{N P}}^{\top} P_{h_{N P}}+\nu^{-2} P_{h 2_{N P}}^{\top}\left[\begin{array}{ccccc}
\varsigma_{1}-\frac{L_{h}}{2} & 0 & \cdots & \cdots & 0 \\
0 & \ddots & \ddots & & \vdots \\
\vdots & \ddots & \ddots & \ddots & \vdots \\
\vdots & & \ddots & \ddots & 0 \\
0 & \cdots & \cdots & 0 & \varsigma_{N}-\frac{L_{h}}{2}
\end{array}\right] P_{h 2_{N P}}\right) \in \mathbb{R}^{n c}
\end{aligned}
$$


with $M_{v q} \in \mathbb{R}^{3 \times n c}, M_{\omega q} \in \mathbb{R}^{3 \times n c}$, and

$$
\begin{gathered}
P_{h 2_{N P}} \triangleq\left[\begin{array}{cccc}
\mathbb{I}_{N-1} & & 0_{N-1 \times 1} \\
-\mathbb{1}_{1 \times N-1} & 0
\end{array}\right] \\
P_{h_{N P}} \triangleq\left[\begin{array}{ccccc}
\nu & 0 & \cdots & 0 & 1 \\
\nu & \ddots & \ddots & \vdots & \vdots \\
\vdots & \ddots & \ddots & 0 & 1 \\
\nu & \cdots & \nu & \nu & 1 \\
0 & \cdots & 0 & 0 & 1
\end{array}\right] .
\end{gathered}
$$

The potential energy of the $N \mathrm{P}$ system is written as

$$
\begin{aligned}
V_{N P} & =\mathbf{z}^{\top} \cdot\left(g \rho \sum_{i=1}^{N} \int_{-\frac{L_{h}}{2}}^{\varsigma_{i}} A_{t}(\sigma)\left(R(\Theta) R_{z}\left(\alpha_{i}\right) \mathbf{r}^{b}(\sigma)+\mathbf{x}^{n}\right) \mathrm{d} \sigma\right) \\
& =-g m_{t} z-g \rho \mathbf{z}^{\top} R(\Theta)\left[\rho \sum_{i=1}^{N} \int_{-\frac{L_{h}}{2}}^{\varsigma_{i}} A_{t}(\sigma) R_{z}\left(\alpha_{i}\right) \mathbf{r}^{b}(\sigma) \mathrm{d} \sigma\right]
\end{aligned}
$$

\section{Appendix B. Derivation of system dynamics}

\section{Appendix B.1. Preliminary results}

For our calculation, we need the following results:

We define the derivative of row vector $x^{\top} \triangleq\left[\begin{array}{lll}x_{1} & \ldots & x_{n}\end{array}\right]$ by column vector $y \triangleq\left[\begin{array}{c}y_{1} \\ \vdots \\ y_{m}\end{array}\right]$ as

$$
\frac{\partial x^{\top}}{\partial y} \triangleq\left[\begin{array}{ccc}
\frac{\partial x_{1}}{\partial y_{1}} & \cdots & \frac{\partial x_{n}}{\partial y_{1}} \\
\vdots & \ddots & \vdots \\
\frac{\partial x_{1}}{\partial y_{m}} & \cdots & \frac{\partial x_{n}}{\partial y_{m}}
\end{array}\right]
$$

Proposition 1. $\forall \mathbf{r} \in \mathbb{R}^{3}$, the derivative of $\mathbf{r}^{\top} R$ by $\Theta$ is given as

$$
\frac{\partial \mathbf{r}^{\top} R}{\partial \Theta}=-G^{\top} S\left(R^{\top} \mathbf{r}\right)
$$

with $G$ as defined in (??), and $S(\cdot)$ is the matrix associated with the crossproduct.

Proof. We detail the calculus for each of the tree base vectors $(\mathbf{x}, \mathbf{y}, \mathbf{z})$. We have

$$
R^{\top} \mathbf{z}=\left[\begin{array}{c}
-s_{\theta} \\
s_{\varphi} c_{\theta} \\
c_{\varphi} c_{\theta}
\end{array}\right]
$$

so

$$
-G^{\top} S\left(R^{\top} \mathbf{z}\right)=\left[\begin{array}{ccc}
0 & c_{\theta} c_{\varphi} & -c_{\theta} s_{\varphi} \\
-c_{\theta} & -s_{\theta} s_{\varphi} & -s_{\theta} c_{\varphi} \\
0 & 0 & 0
\end{array}\right]
$$


and

$$
\frac{\partial \mathbf{z}^{\top} R}{\partial \Theta}=\left[\begin{array}{ccc}
0 & c_{\theta} c_{\varphi} & -c_{\theta} s_{\varphi} \\
-c_{\theta} & -s_{\theta} s_{\varphi} & -s_{\theta} c_{\varphi} \\
0 & 0 & 0
\end{array}\right]
$$

Therefore,

$$
\frac{\partial \mathbf{z}^{\top} R}{\partial \Theta}=-G^{\top} S\left(R^{\top} \mathbf{z}\right) .
$$

We also have

$$
R^{\top} \mathbf{y}=\left[\begin{array}{c}
c_{\theta} s_{\psi} \\
c_{\varphi} c_{\psi}+s_{\varphi} s_{\theta} s_{\psi} \\
-s_{\varphi} c_{\psi}+c_{\varphi} s_{\theta} s_{\psi}
\end{array}\right]
$$

so,

$$
-G^{\top} S\left(R^{\top} \mathbf{y}\right)=\left[\begin{array}{ccc}
0 & -s_{\varphi} c_{\psi}+c_{\varphi} s_{\theta} s_{\psi} & -c_{\varphi} c_{\psi}-s_{\varphi} s_{\theta} s_{\psi} \\
-s_{\theta} s_{\psi} & s_{\varphi} c_{\theta} s_{\psi} & c_{\varphi} c_{\theta} s_{\psi} \\
c_{\theta} c_{\psi} & -c_{\varphi} s_{\psi}+s_{\varphi} s_{\theta} c_{\psi} & s_{\varphi} s_{\psi}+c_{\varphi} s_{\theta} c_{\psi}
\end{array}\right]
$$

and

$$
\frac{\partial \mathbf{y}^{\top} R}{\partial \Theta}=\left[\begin{array}{ccc}
0 & -s_{\varphi} c_{\psi}+c_{\varphi} s_{\theta} s_{\psi} & -c_{\varphi} c_{\psi}-s_{\varphi} s_{\theta} s_{\psi} \\
-s_{\theta} s_{\psi} & s_{\varphi} c_{\theta} s_{\psi} & c_{\varphi} c_{\theta} s_{\psi} \\
c_{\theta} c_{\psi} & -c_{\varphi} s_{\psi}+s_{\varphi} s_{\theta} c_{\psi} & s_{\varphi} s_{\psi}+c_{\varphi} s_{\theta} c_{\psi}
\end{array}\right]
$$

Therefore,

$$
\frac{\partial \mathbf{y}^{\top} R}{\partial \Theta}=-G^{\top} S\left(R^{\top} \mathbf{y}\right)
$$

Finally,

$$
R^{\top} \mathbf{x}=\left[\begin{array}{c}
c_{\theta} c_{\psi} \\
-c_{\varphi} s_{\psi}+s_{\varphi} s_{\theta} c_{\psi} \\
s_{\varphi} s_{\psi}+c_{\varphi} s_{\theta} c_{\psi}
\end{array}\right]
$$

so,

$$
-G^{\top} S\left(R^{\top} \mathbf{x}\right)=\left[\begin{array}{ccc}
0 & s_{\varphi} s_{\psi}+c_{\varphi} s_{\theta} c_{\psi} & c_{\varphi} s_{\psi}-s_{\varphi} s_{\theta} c_{\psi} \\
-s_{\theta} c_{\psi} & s_{\varphi} c_{\theta} c_{\psi} & c_{\varphi} c_{\theta} c_{\psi} \\
-c_{\theta} s_{\psi} & -c_{\varphi} c_{\psi}-s_{\varphi} s_{\theta} s_{\psi} & s_{\varphi} c_{\psi}-c_{\varphi} s_{\theta} s_{\psi}
\end{array}\right]
$$

and

$$
\frac{\partial \mathbf{x}^{\top} R}{\partial \Theta}=\left[\begin{array}{ccc}
0 & s_{\varphi} s_{\psi}+c_{\varphi} s_{\theta} c_{\psi} & c_{\varphi} s_{\psi}-s_{\varphi} s_{\theta} c_{\psi} \\
-s_{\theta} c_{\psi} & s_{\varphi} c_{\theta} c_{\psi} & c_{\varphi} c_{\theta} c_{\psi} \\
-c_{\theta} s_{\psi} & -c_{\varphi} c_{\psi}-s_{\varphi} s_{\theta} s_{\psi} & s_{\varphi} c_{\psi}-c_{\varphi} s_{\theta} s_{\psi}
\end{array}\right]
$$

Therefore,

$$
\frac{\partial \mathbf{x}^{\top} R}{\partial \Theta}=-G^{\top} S\left(R^{\top} \mathbf{x}\right) .
$$

With $\mathbf{r}=r_{1} \mathbf{x}+r_{2} \mathbf{y}+r_{3} \mathbf{z}$, by linearity,

$$
\frac{\partial \mathbf{r}^{\top} R}{\partial \Theta}=-G^{\top} S\left(R^{\top} \mathbf{r}\right) .
$$


Proposition 2. The derivatives of $\mathbf{v}^{b \top}$ and $\omega^{b \top}$ by $\Theta$ are

$$
\begin{aligned}
& \frac{\partial \mathbf{v}^{b \top}}{\partial \Theta}=-G^{\top} S\left(\mathbf{v}^{b}\right) \\
& \frac{\partial \omega^{b \top}}{\partial \Theta}=\dot{G}^{\top}-G^{\top} S\left(\omega^{b}\right) .
\end{aligned}
$$

Proof. Hence,

$$
\omega^{b}=G(\Theta) \dot{\Theta}=\left[\begin{array}{c}
\dot{\varphi}+s_{\theta} \dot{\psi} \\
c_{\theta} s_{\varphi} \dot{\psi}+c_{\varphi} \dot{\theta} \\
c_{\theta} c_{\varphi} \dot{\psi}-s_{\varphi} \dot{\theta}
\end{array}\right]
$$

so,

$$
\frac{\partial \omega^{b \top}}{\partial \Theta}=\left[\begin{array}{ccc}
0 & c_{\theta} c_{\varphi} \dot{\psi}-s_{\varphi} \dot{\theta} & -c_{\theta} s_{\varphi} \dot{\psi}-c_{\varphi} \dot{\theta} \\
-c_{\theta} \dot{\psi} & -s_{\theta} s_{\varphi} \dot{\psi} & -s_{\theta} c_{\varphi} \dot{\psi} \\
0 & 0 & 0
\end{array}\right]
$$

With $G$ as defined in (??),

$$
\dot{G}^{\top}=\left[\begin{array}{ccc}
0 & 0 & 0 \\
0 & -s_{\varphi} \dot{\varphi} & -c_{\varphi} \dot{\varphi} \\
-c_{\theta} \dot{\theta} & c_{\theta} c_{\varphi} \dot{\varphi}-s_{\theta} s_{\varphi} \dot{\theta} & -c_{\theta} s_{\varphi} \dot{\varphi}-s_{\theta} c_{\varphi} \dot{\theta}
\end{array}\right] .
$$

We also write

$$
G^{\top} S(G(\Theta) \dot{\Theta})=\left[\begin{array}{ccc}
0 & -c_{\theta} c_{\varphi} \dot{\psi}+s_{\varphi} \dot{\theta} & c_{\theta} s_{\varphi} \dot{\psi}+c_{\varphi} \dot{\theta} \\
c_{\theta} \dot{\psi} & s_{\varphi}\left(-\dot{\varphi}+s_{\theta} \dot{\psi}\right) & c_{\varphi}\left(-\dot{\varphi}+s_{\theta} \dot{\psi}\right) \\
-c_{\theta} \dot{\theta} & c_{\theta} c_{\varphi} \dot{\varphi}-s_{\theta} s_{\varphi} \dot{\theta} & -c_{\theta} s_{\varphi} \dot{\varphi}-s_{\theta} c_{\varphi} \dot{\theta}
\end{array}\right] .
$$

Therefore,

$$
\frac{\partial \omega^{b \top}}{\partial \Theta}=\dot{G}^{\top}-G^{\top} S\left(\omega^{b}\right)
$$

As $\mathbf{v}^{b}$ is $R^{\top} \dot{\mathbf{x}}^{e}$, according to Proposition 1,

$$
\frac{\partial \mathbf{v}^{b \top}}{\partial \Theta}=-G^{\top} S\left(\mathbf{v}^{b}\right) \text {. }
$$

Appendix B.2. Derivation of the dynamics for the $N U$ system

Using a Lagrangian approach, the dynamics of the system are given by

$$
\frac{\mathrm{d}}{\mathrm{d} t} \frac{\partial\left(T_{N U}-V_{N U}\right)}{\partial \dot{q}}-\frac{\partial\left(T_{N U}-V_{N U}\right)}{\partial q}=Q
$$

We first derive $\frac{\partial T_{N U}}{\partial q}$. According to (A.1), $T$ is independent of $x^{n}$; therefore,

$$
\frac{\partial T_{N U}}{\partial x^{n}}=0_{3 \times 1} .
$$

As $M$ is symmetrical and is not a function of $\Theta$,

$$
\frac{\partial T_{N U}}{\partial \Theta}=\frac{1}{2} \frac{\partial\left(\dot{q}^{\top} \mathcal{P}^{\top} M_{N U} \mathcal{P} \dot{q}\right)}{\partial \Theta}=\frac{\partial(\mathcal{P} \dot{q})^{\top}}{\partial \Theta} M_{N U}(\mathcal{P} \dot{q}) .
$$


Using (B.3) and (B.4),

$$
\frac{\partial(\mathcal{P} \dot{q})^{\top}}{\partial \Theta}=\frac{\partial v^{\top}}{\partial \Theta}=\left[\frac{\partial \mathbf{v}^{b \top}}{\partial \Theta}, \frac{\partial \omega^{b \top}}{\partial \Theta}, 0_{3 \times 1}\right]=\left[\begin{array}{lll}
-G^{\top} S\left(\mathbf{v}^{b}\right) & \dot{G}^{\top}-G^{\top} S\left(\omega^{b}\right) & 0_{3 \times 1}
\end{array}\right] .
$$

The term $\frac{\partial T}{\partial w_{i}}$ can be expressed as

$$
\frac{\partial T_{N U}}{\partial w_{i}}=\frac{1}{2} \dot{q}^{\top} \mathcal{P}^{\top} \frac{\partial M_{N U}}{\partial w_{i}} \mathcal{P} \dot{q}
$$

with

$$
\begin{aligned}
\frac{\partial}{\partial w_{i}} M_{v \omega} & =-\rho A_{v} S\left(R_{z}\left(\alpha_{i}\right) \mathbf{r}^{b}\left(\varsigma_{p i}\right)-R_{z}\left(\alpha_{i}\right) \mathbf{r}^{b}\left(-\varsigma_{s i}\right)\right) \\
\frac{\partial}{\partial w_{i}} M_{\omega} & =-\rho A_{v}\left(S\left(R_{z}\left(\alpha_{i}\right) \mathbf{r}^{b}\left(\varsigma_{p i}\right)\right)^{2}-S\left(R_{z}\left(\alpha_{i}\right) \mathbf{r}^{b}\left(-\varsigma_{s i}\right)\right)^{2}\right) \\
\frac{\partial}{\partial w_{i}} M_{v q}[:, i] & =\rho A_{v}\left(R_{z}\left(\alpha_{i}\right) \frac{\mathrm{d} \mathbf{r}^{b}}{\mathrm{~d} \sigma}\left(\varsigma_{p i}\right)-R_{z}\left(\alpha_{i}\right) \frac{\mathrm{d} \mathbf{r}^{b}}{\mathrm{~d} \sigma}\left(-\varsigma_{s i}\right)\right) \\
\frac{\partial}{\partial w_{i}} M_{v q}[:, j \neq i] & =0_{3 \times 1} \\
\frac{\partial}{\partial w_{i}} M_{\omega q}[:, i] & =\rho A_{v}\left(S\left(R_{z}\left(\alpha_{i}\right) \mathbf{r}^{b}\left(\varsigma_{p i}\right)\right) R_{z}\left(\alpha_{i}\right) \frac{\mathrm{d} \mathbf{r}^{b}}{\mathrm{~d} \sigma}\left(\varsigma_{p i}\right)-S\left(R_{z}\left(\alpha_{i}\right) \mathbf{r}^{b}\left(-\varsigma_{s i}\right)\right) R_{z}\left(\alpha_{i}\right) \frac{\mathrm{d} \mathbf{r}^{b}}{\mathrm{~d} \sigma}\left(-\varsigma_{s i}\right)\right) \\
\frac{\partial}{\partial w_{i}} M_{\omega q}[:, j \neq i] & =0_{3 \times 1} \\
\frac{\partial}{\partial w_{i}} M_{q} & =0_{n c \times n c} .
\end{aligned}
$$

According to (5), and (B.2), $\frac{\partial V_{N U}}{\partial q}$ is given by

$$
\begin{aligned}
& \frac{\partial V_{N U}}{\partial x^{n}}=\left[\begin{array}{c}
0 \\
0 \\
-g m_{t}
\end{array}\right]=-g m_{t} \mathbf{z} \\
& \frac{\partial V_{N U}}{\partial \Theta}=-g \rho \frac{\partial \mathbf{z}^{\top} R}{\partial \Theta}\left(\sum_{i=1}^{n c} \int_{-\varsigma_{s i}}^{\varsigma_{p i}} A_{t}(\sigma) R_{z}\left(\alpha_{i}\right) \mathbf{r}^{b}(\sigma) \mathrm{d} \sigma\right) \\
& =g \rho G^{\top} S\left(R^{\top} \mathbf{z}\right)\left(\sum_{i=1}^{n c} \int_{-\varsigma_{s i}}^{\varsigma_{p i}} A_{t}(\sigma) R_{z}\left(\alpha_{i}\right) \mathbf{r}^{b}(\sigma) \mathrm{d} \sigma\right) \\
& =g \rho G^{\top} S\left(R^{\top} \mathbf{z}\right)\left(A_{h} \sum_{i=1}^{n c}\left[\begin{array}{c}
\nu L_{h} w_{i} \sin \alpha_{i} \\
-\nu L_{h} w_{i} \cos \alpha_{i} \\
\nu\left(2 L_{v} e-L_{v}^{2}-w_{i}^{2}\right)+L_{h} e
\end{array}\right]\right) \\
& \frac{\partial V_{N U}}{\partial w_{i}}=-g \rho A_{v} \mathbf{z}^{\top} R(\Theta) R_{z}\left(\alpha_{i}\right)\left(\mathbf{r}^{b}\left(\varsigma_{p i}\right)-\mathbf{r}^{b}\left(-\varsigma_{s i}\right)\right) .
\end{aligned}
$$

According to (5), $V_{N U}$ is not a function of $\dot{q}$; thus,

$$
\frac{\mathrm{d}}{\mathrm{d} t} \frac{\partial V_{N U}}{\partial \dot{q}}=0_{6+n c \times 1} .
$$

We also have

$$
\frac{\mathrm{d}}{\mathrm{d} t}\left(\frac{\partial T_{N U}}{\partial \dot{q}}\right)^{\top}=\mathcal{M}_{N U} \ddot{q}+\left(\dot{\mathcal{P}}^{\top} M_{N U} \mathcal{P}+\mathcal{P}^{\top} \sum_{i=1}^{n c} \dot{w}_{i} \frac{\partial M_{N U}}{\partial w_{i}} \mathcal{P}+\mathcal{P}^{\top} M_{N U} \dot{\mathcal{P}}\right) \dot{q}
$$


Appendix B.3. Summary of $N U$ system dynamics

We write the dynamics of the system as

$$
\mathcal{M}_{N U}(q) \ddot{q}+C_{N U}(q, \dot{q}) \dot{q}+k_{N U}(q)=Q_{h y d r o}(t, \beta)+Q_{r^{2} s_{N U}}(\dot{w})
$$

with $\mathcal{M}_{N U}(q)$ defined in (A.1), and

$$
\begin{gathered}
C_{N U} \triangleq \dot{\mathcal{P}}^{\top} M_{N U} \mathcal{P}+\mathcal{P}^{\top} \sum_{i=1}^{n c} \dot{w}_{i} \frac{\partial M_{N U}}{\partial w_{i}} \mathcal{P}+\mathcal{P}^{\top} M_{N U} \dot{\mathcal{P}}-\left[\begin{array}{c}
0_{3 \times 6+n c} \\
\frac{\partial(\mathcal{P} \dot{q})^{\top}}{\partial^{\top}} M_{N U} \mathcal{P} \\
\frac{1}{2} \dot{q}^{\top} \mathcal{P}^{\top} \frac{\partial M_{N U}}{\partial w_{1}} \mathcal{P} \\
\vdots \\
\frac{1}{2} \dot{q}^{\top} \mathcal{P}^{\top} \frac{\partial M_{N U}}{\partial w_{n c}} \mathcal{P}
\end{array}\right] \\
k_{N U} \triangleq-g\left[\begin{array}{c}
-\rho G^{\top} S\left(R^{\top} \mathbf{z}\right) \sum_{i=1}^{n c} \int_{-\varsigma_{s i}}^{\varsigma_{p i} \mathbf{z}} A_{t}(\sigma) R_{z}\left(\alpha_{i}\right) \mathbf{r}^{b}(\sigma) \mathrm{d} \sigma \\
\rho A_{v} \mathbf{z}^{\top} R(\Theta) R_{z}\left(\alpha_{1}\right)\left(\mathbf{r}^{b}\left(\varsigma_{p 1}\right)-\mathbf{r}^{b}\left(-\varsigma_{s 1}\right)\right) \\
\vdots \\
\rho A_{v} \mathbf{z}^{\top} R(\Theta) R_{z}\left(\alpha_{n c}\right)\left(\mathbf{r}^{b}\left(\varsigma_{p n c}\right)-\mathbf{r}^{b}\left(-\varsigma_{s n c}\right)\right)
\end{array}\right] \\
Q_{r e s_{N U}}=\left[\begin{array}{c}
0_{1 \times 6} \\
P_{h_{N U}}^{\top} F_{h}(\dot{w})
\end{array}\right] \\
P_{h_{N U}}=\nu \mathbb{I}_{n c} .
\end{gathered}
$$

Appendix B.4. Summary of NS system dynamics

Using the expressions of the energies obtained in Appendix A.2, and following the method used in Appendix B for the $N \mathrm{U}$ system, we write the dynamics of the NS system as

$$
\mathcal{M}_{N S}(q) \ddot{q}+C_{N S}(q, \dot{q}) \dot{q}+k_{N S}(q)=Q_{h y d r o}(t, \beta)+Q_{r e s_{N S}}(\dot{w})
$$

with

$$
\begin{aligned}
& \mathcal{M}_{N S}(q) \triangleq \mathcal{P}(\Theta)^{\top} M_{N S}(w) \mathcal{P}(\Theta) \\
& C_{N S} \triangleq \dot{\mathcal{P}}^{\top} M_{N S} \mathcal{P}+\mathcal{P}^{\top} \sum_{i=1}^{n c} \dot{w}_{i} \frac{\partial M_{N S}}{\partial w_{i}} \mathcal{P}+\mathcal{P}^{\top} M_{N S} \dot{\mathcal{P}}-\left[\begin{array}{c}
03 \times 6+n c \\
\frac{\partial(\mathcal{P} \dot{q})^{\top}}{\partial{ }^{\top}} M_{N S} \mathcal{P} \\
\frac{1}{2} \dot{q}^{\top} \mathcal{P}^{\top} \frac{\partial M_{N S}}{\partial w_{1}} \mathcal{P} \\
\vdots \\
\frac{1}{2} \dot{q}^{\top} \mathcal{P}^{\top} \frac{\partial M_{N S}}{\partial w_{n c}} \mathcal{P}
\end{array}\right] \\
& k_{N S} \triangleq-g\left[\begin{array}{c}
m_{t} \mathbf{z} \\
-\rho G^{\top} S\left(R^{\top} \mathbf{z}\right) \sum_{i=1}^{N} \int_{0}^{\varsigma_{i}} A_{t}(\sigma) R_{z}\left(\alpha_{i}\right) \mathbf{r}^{b}(\sigma) \mathrm{d} \sigma \\
\rho A_{v} \mathbf{z}^{\top} R(\Theta)\left(R_{z}\left(\alpha_{1}\right) \mathbf{r}^{b}\left(\varsigma_{1}\right)-R_{z}\left(\alpha_{N}\right) \mathbf{r}^{b}\left(\varsigma_{N}\right)\right) \\
\vdots \\
\rho A_{v} \mathbf{z}^{\top} R(\Theta)\left(R_{z}\left(\alpha_{n c}\right) \mathbf{r}^{b}\left(\varsigma_{n c}\right)-R_{z}\left(\alpha_{N}\right) \mathbf{r}^{b}\left(\varsigma_{N}\right)\right)
\end{array}\right] \\
& Q_{r e s_{N S}}=\left[\begin{array}{c}
0_{1 \times 6} \\
P_{h_{N S}}^{\top} F_{h}(\dot{w})
\end{array}\right] \\
& P_{h_{N S}}=\nu\left[\begin{array}{c}
\mathbb{I}_{n c} \\
-\mathbb{1}_{1 \times n c}
\end{array}\right] \text {. }
\end{aligned}
$$


Appendix B.5. Summary of NP system dynamics

Using the expressions of the energies obtained in Appendix A.3, and following the method used in Appendix B for the NU system, we write the dynamics of the NP system as

$$
\mathcal{M}_{N P}(q) \ddot{q}+C_{N P}(q, \dot{q}) \dot{q}+k_{N P}(q)=Q_{h y d r o}+Q_{r e s_{N P}}(\dot{w})
$$

with

$$
\begin{aligned}
& \mathcal{M}_{N P}(q) \triangleq \mathcal{P}(\Theta)^{\top} M_{N P}(w) \mathcal{P}(\Theta) \\
& C_{N P} \triangleq \dot{\mathcal{P}}^{\top} M_{N P} \mathcal{P}+\mathcal{P}^{\top} \sum_{i=1}^{n c} \dot{w}_{i} \frac{\partial M_{N P}}{\partial w_{i}} \mathcal{P}+\mathcal{P}^{\top} M_{N P} \dot{\mathcal{P}}-\left[\begin{array}{c}
0_{3 \times 6+n c} \\
\frac{\partial(\mathcal{P} \dot{q})^{\top}}{\partial{ }^{\top}} M_{N P} \mathcal{P} \\
\frac{1}{2} \dot{q}^{\top} \mathcal{P}^{\top} \frac{\partial M_{N P} \mathcal{P}}{\partial w_{1}} \\
\vdots \\
\frac{1}{2} \dot{q}^{\top} \mathcal{P}^{\top} \frac{\partial M_{N P}}{\partial w_{n c-1}} \mathcal{P} \\
0_{1 \times 6+n c}
\end{array}\right] \\
& k_{N P} \triangleq-g\left[\begin{array}{c}
m_{t} \mathbf{z} \\
-\rho G^{\top} S\left(R^{\top} \mathbf{z}\right) \sum_{i=1}^{N} \int_{-\frac{L_{h}}{2}}^{\varsigma_{i}} A_{t}(\sigma) R_{z}\left(\alpha_{i}\right) \mathbf{r}^{b}(\sigma) \mathrm{d} \sigma \\
\rho A_{v} \mathbf{z}^{\top} R(\Theta)\left(R_{z}\left(\alpha_{1}\right) \mathbf{r}^{b^{2}}\left(\varsigma_{1}\right)-R_{z}\left(\alpha_{N}\right) \mathbf{r}^{b}\left(\varsigma_{N}\right)\right) \\
\vdots \\
\rho A_{v} \mathbf{z}^{\top} R(\Theta)\left(R_{z}\left(\alpha_{N-1}\right) \mathbf{r}^{b}\left(\varsigma_{N-1}\right)-R_{z}\left(\alpha_{N}\right) \mathbf{r}^{b}\left(\varsigma_{N}\right)\right) \\
0
\end{array}\right] \\
& Q_{r e s_{N P}}=\left[\begin{array}{c}
0_{1 \times 6} \\
P_{h_{N P}}^{\top} F_{h}(\dot{w})
\end{array}\right] \\
& P_{h_{N P}}=\left[\begin{array}{ccccc}
\nu & 0 & \cdots & 0 & 1 \\
\nu & \ddots & \ddots & \vdots & \vdots \\
\vdots & \ddots & \ddots & 0 & 1 \\
\nu & \cdots & \nu & \nu & 1 \\
0 & \cdots & 0 & 0 & 1
\end{array}\right] \text {. }
\end{aligned}
$$

